NASA Technical Memorandum 101417

AIAA-89-0437

\title{
Numerical Analysis of Flow Through Oscillating Cascade Sections
}

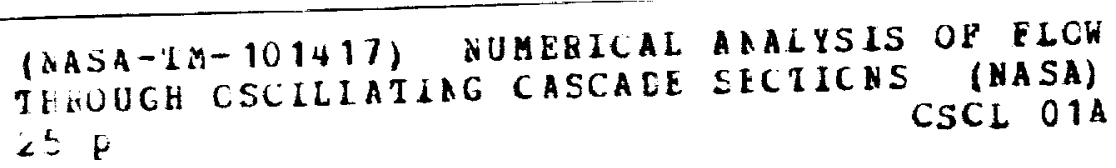

Dennis L. Huff

Lewis Research Center

Cleveland, Ohio

Prepared for the 27th Aerospace Sciences Meeting sponsored by the American Institute of Aeronautics and Astronautics Reno, Nevada, January 9-12, 1989 
$\cap \Omega$ 


\title{
NUMERICAL ANALYSIS OF FLOW THROUGH OSCILLATING CASCADE SECTIONS
}

\author{
Dennis L. Huff \\ National Acronautics and Space Administration \\ Lewis Research Center \\ Cleveland, Ohio 44135
}

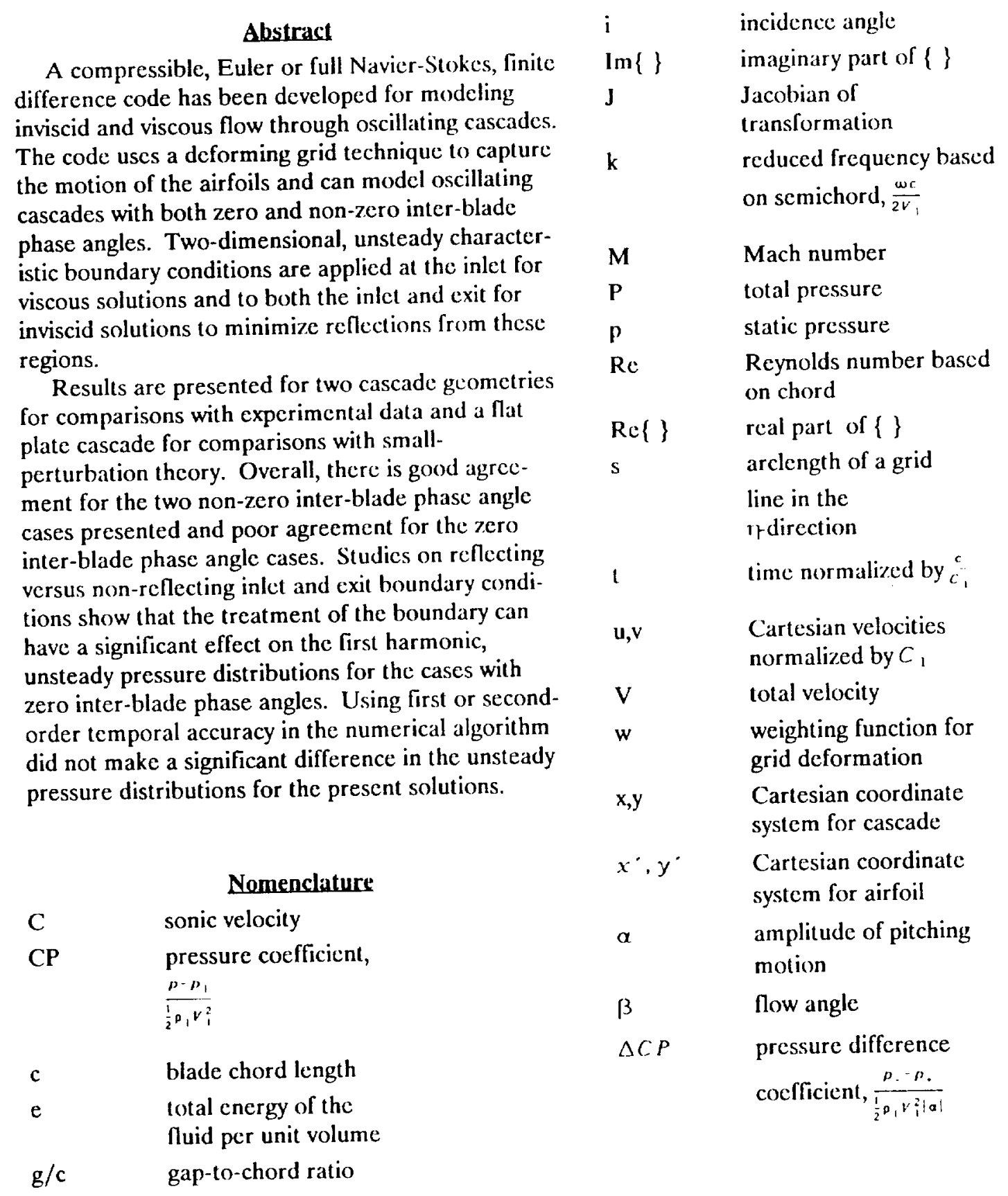

This paper is declared a work of the U.S. Government and is not subject to copyright protection in the United States. 


\begin{tabular}{|c|c|}
\hline$\triangle C P$ & $\begin{array}{l}\text { pressure difference } \\
\text { coefficient, } \frac{p, n}{p_{1}, v_{i}|a|}\end{array}$ \\
\hline$\gamma$ & stagger angle \\
\hline$\eta$ & $\begin{array}{l}\text { normal direction of } \\
\text { transformed coordinate } \\
\text { system }\end{array}$ \\
\hline$\xi$ & $\begin{array}{l}\text { chordwise dircetion of } \\
\text { transformed coordinate } \\
\text { system }\end{array}$ \\
\hline$\phi$ & $\begin{array}{l}\text { phase lead angle } \\
\text { relative to the } \\
\text { complex angular } \\
\text { displacement }\end{array}$ \\
\hline$\rho$ & fluid density \\
\hline$\tau$ & time variable \\
\hline$\tau^{\prime}$ & $\begin{array}{l}\text { thickness-to-chord } \\
\text { ratio }\end{array}$ \\
\hline$\sigma$ & inter-blade phase angle \\
\hline$\omega$ & $\begin{array}{l}\text { airfoil oscillation } \\
\text { frequency }\end{array}$ \\
\hline 1 & conditions at the inlet \\
\hline 2 & conditions at the exit \\
\hline,+- & $\begin{array}{l}\text { upper and lower } \\
\text { surfaces on airfoil, } \\
\text { respectively }\end{array}$ \\
\hline
\end{tabular}

\section{Introduction}

The design of turbomachinery blades requires the prevention of flutter for all operaling conditions. However, flow field predictions used for aeroelastic analysis are not well developed for all now regimes. Many methods used for flutter analysis depend on the successful predictions of blade loading and blade motion. Ideally, this is an interactive process where a structural analysis determines the blade motion from the blade loading and the loading is determine by the now analysis from the blade motion. Unfortunatcly, the flow analysis technology lags the structural analysis technology in that accurate predictions of the flow field for transonic flow through oscillating cascades are not readily available. Many of the existing now analysis methods are numerical and require large amounts of computer time. However, advances in computer technology have given the acrodynamicist hope for numerical methods being used as a tool in the design process.

In theory, the solution of the Navicr-Stokes equations should model all flow phenomena associated with transonic flow through oscillating cascades. Since exact solutions are not available, computational techniques are used and introduce approximations to the solutions. These approximations commonly use eddy viscosity methods for turbulence modeling, numerical dissipation terms for stability and coarse grids for practical computing. Further complications arise when unsteady flow exists and proper treatment of the boundary conditions are essential. The message here is that numerical solutions of the more complex flow equations, like the Navier-Stokes equations, do not automatically give accurate results. Much research is needed in this area to develop proper modeling of the physical flow ficld.

The present research focuses on numerical solutions of the Euler and Navier-Stokes equations to modcl two-dimensional flow through oscillating cascades. The model prescribes harmonic pitching motions for the blade sections for both zero and non-zero inter-blade phase angles. The deforming grid technique introduced in reference 1 is utilized for convenient specification of the periodic boundary conditions. Several sample predictions are compared to expcrimental data. Also, an investigation of reflecting versus non-reflecting boundary conditions is presented to determine their effect on the unsteady blade loading.

\section{Governing Equations}

A major portion of the present code is based on the unsteady, viscous solver developed by Sankar and Tang (ref. 2) for flow past isolated airfoils. This code solves the two-dimensional, unsteady, Reynoldsaveraged, compressible Navier-Stokes equations on a body-fitted moving coordinate system in strong conservation form using an ADI procedure. These equations can be written as:

$$
\bar{q}_{\mathrm{t}}+F_{\eta}+\bar{C}_{n}=R_{t}+\hat{S}_{\eta}
$$


where

$$
\hat{q}=J^{-1}\{\rho, \rho u, \rho u, e\}
$$

and $\rho$ is the fluid density; $u$ and $v$ are the Cartesian components of the fluid velocity; $\mathrm{c}$ is the total energy per unit volume. The body-fitted $(\xi, \eta, \tau)$ coordinate system is related to the Cartesian coordinates using the following transformation:

$$
\begin{gathered}
\xi=\xi(x, y, 1) \\
\eta=\eta(x, y, 1) \\
\tau=t
\end{gathered}
$$

The Jacobian of the transformation is given by:

$$
J=\xi_{x} \eta_{y}-\eta_{x} \xi_{x y}=\frac{1}{x_{y} y_{11}-x_{11} y_{1}}
$$

and the metries of the transformation are given by:

$$
\begin{array}{ll}
\xi_{x}=J y_{\eta} ; & \eta_{x}=-J y_{\xi} \\
\xi_{y}=-J x_{\eta} ; & \eta_{y}=J x_{\xi}
\end{array}
$$

Standard central differences were used lo compute $x_{\xi}, y_{\xi}, x_{\eta}, y_{n}$ which were then used to calculate the metrics.

The $f$ and $\xi$ terms in equation (1) are the inviscid terms in the $\xi$ and $r$-directions, respectively. The viscous terms, $R$ and $S$, are treated explicitly and can be omitted to give solutions for the Euler cquations. The Beam-Warming, block ADI algorithm is used to solve the governing equations. Artificial dissipation is added to help the stability. The solution is secondorder accurate in space and first or second-order accurate in time. The Baldwin-Lomax, two-layer algebraic model (ref. 3 ) is used to cvaluate the eddy viscosity. The Johnson-King (ref. 4) and Gorski (ref. 5) $k-\epsilon$ turbulence models have been coded by $W u$ (ref. 6), but no comparisons are included in this study. Further information about the algorithm for isolated airfoils can be found in reference 2 .

\section{Grid}

An unique feature of the present code is the treatment of the grid for oscillating cascades. A method for deforming the grid was developed in reference 1 for zero inter-blade phase angles. The present study is an extension of this method to model non-zero inter-blade phase angles.

The code uses a C-grid generated from Sorenson's (rcl. 7) GRAPE code, which was modified by Chima (ref. 8) for improved modeling in turbomachinery problems. One C-grid is gencrated for each blade in the cascade. The outer boundary of the $\mathrm{C}$-grid is defined by the user in the GRAPE code. A deforming grid technique (ref. 1) is used to locate the position of the grid as a function of time. The inner boundary moves with the prescribed blade motion, while the outer boundary remains fixed in space. The grid lines connecting the inner and outer boundaries are allowed to deform. The amount of deformation is a function of the distance away from the surface of the airfoil. A wcighting function, $w$, is defined as:

$$
w_{11}=w(\xi, \eta)=\left|\frac{s(\xi, \eta)}{s\left(\xi, \eta_{\max }\right)}-1\right|
$$

where $s=$ the arclength of a grid line from the airfoil surface $(\eta=1)$ to some grid point along $\xi=$ constant, and $\eta_{\max }=$ the outer boundary grid linc. The grid deformation is defined as:

$$
\begin{aligned}
& \Delta x_{1 j}=w_{11}\left(\Delta x_{1 j}\right) \\
& \Delta y_{1 j}=w_{1 j}\left(\Delta y_{1 j}\right)
\end{aligned}
$$

where $\Delta x_{1 /}$ and $\Delta y_{1 /}$ are the spatial differences between successive time steps if the entire grid was moved as a rigid body. From equations (6) and (7), we see that nodes at the inner boundary $(s=0)$ gives $u_{11}=1$, which means the airfoil surface follows the rigid body motion of the blade. Conversely, the outer boundary nodes give $w_{11}=0$ and the node positions remain fixed at the initial specified locations. The interior nodes shear in space relative to the initial grid as $w_{1}$, varies between 0 and 1 . The node velocities can be easily found by dividing the grid deformation by the time step value.

Multiple blade computations are made possible by stacking the $\mathrm{C}$-grids for each blade and passing information between the upper and lower boundaries. 
Each C-grid is expanded by one grid line in the rodirection at the outer boundary to provide ghost points for the interior grid of the adjacent blades. This allows the periodic boundary condition to be treated implicilly. The case for zero inter-blado phase angle is the simplest for grid generation. Periodic boundary conditions are applied across the upper and lower boundaries thereby requiring a grid for only one blade. However, non-zero inter-blade phase angles require multiple blade computations for exact treatment of the periodic boundary conditions. For example; an inter-blade phase angle (c) of \pm 180 degrees requires two blades, $\sigma= \pm 90$ degrees requires four blades, etc. This significantly increases the computational time for non-zcro inter-blade phase angles, but provides an exact boundary condition. This method may be useful as a tool for evaluating simplified periodic boundary conditions that do not use as many blades for non-zero inter-blade phase angles.

The deforming grid technique is demonstrated in Figure 1 for a multiple blade computation. The figure shows two extremely simplified grids superimposed at two different times. Multiple grids are generated for a NACA 16-004 cascade with twenty degrees of stagger. This geometry is representative of a geometry that might be used in the advanced turboprop. The inter-blade phase angle is 9 ) degrees and requires four blades to specify the periodic boundary conditions. (Only three blades are shown in this figure for clarity.) The amount of oscillation is exaggerated to illustrate the deforming grid technique. Notice how the outer boundary of the grid around one blade remains fixed in space, while the inner boundary follows the motion of the airfoil. An actual calculation for an oscillating cascade typically has a pitching amplitude of only a few degrees and does not distort the grid as much as shown in Figurc 1. Larger pitching amplitudes are possible, however, by regriding at different time periods and using the deforming grid technique for various time intervals of the pitching motion.

\section{Boundary Conditions}

The proper treatment of boundary conditions is important for any numerical computation and especially for unsteady flows. The present solution solves for the flow around each blade independently and uses periodic boundary conditions along the upper and lower boundaries to model the cascade effects. Ghost points are assigned at the first interior grid line $\left(11=\eta_{\max }-1\right)$ and are used implicitly by the adjacent grid from the next blade. Although it is tempting to use the most current flow information as it becomes available from the integration scheme, it is important to only use flow information from the same time period across the periodic boundaries. This eliminates time inaccuracy due to the direction of time marching from blade to blade. The metric data is also forced to be continuous along the periodic boundaries. This procedure essentially makes the periodic boundaries invisible to the flow solution and means the only specification of boundary conditions are at the inlet and exit planes, blade surfaces and the slits aft of the airfoils.

The inlet conditions are assumed to be uniform with a reference density, velocity and inlet flow angle. In previous solutions (ref. 1), the static pressure was also specificd from the stcady-state solution of a stationary cascade. This provided enough information to determine the unsteady solution for an oscillating cascade, but also introduces a reflective boundary condition. This problem has been addressed by transforming the fluid equations into characteristic form at the inlet boundary and prescribing the flow information as incoming or outgoing waves. This approach was formulated by Hedstrom (ref. 9) for one-dimensional gas dynamic equations, and extended to two-dimensional problems by Thompson (ref. 10). Whilc the two-dimensional method does not give a perfectly transmitting boundary condition, it does offer a method for minimizing reflections by considering waves oblique to the grid boundary. A brief explanation of this method is given below.

The two-dimensional fluid equations from equation (1) can be generalized in the following form if we neglect viscous terms:

$$
\frac{\partial Q}{\partial t}+\frac{\partial F}{\partial \xi}+\frac{\partial G}{\partial \eta}+C_{\xi}+C_{\eta}=0
$$

Define the following relations:

$$
\begin{gathered}
\frac{\partial U}{\partial t}=P \frac{\partial U}{\partial t}, \frac{\partial F}{\partial \xi}=Q \frac{\partial U}{\partial \xi}, \\
\frac{\partial G}{\partial \eta}=R \frac{\partial U}{\partial \eta}, A=P^{-1} Q . B=P^{-1} R,
\end{gathered}
$$




$$
C_{\mathrm{g}}=P^{-1} C_{\mathrm{g}}, C_{\mathrm{n}}=P^{1} C_{\mathrm{n}}^{\prime}
$$

Substituting into equation (8) and multipying by $P^{-1}$ gives:

$$
\frac{\partial U}{\partial t}+A \frac{\partial U}{\partial \xi}+B \frac{\partial U}{\partial \eta}+C_{\xi}+C_{\eta}=0
$$

The $A$ and $B$ matrices can be defined as:

$$
A=S^{-1} \wedge S, B=T^{-1} H T
$$

where $\Lambda$ and $\mathrm{H}$ are diagonal matrices which define the characteristic velocities. Hence, the characteristic form of equation (8) can be written as:

$$
\begin{gathered}
\frac{\partial U}{\partial l}+S^{-1} \\
\wedge S \frac{\partial U}{\partial \xi}+T^{-1} H T \frac{\partial U}{\partial \eta} \\
+C_{\xi}+C_{n}=0
\end{gathered}
$$

A portion of the $\eta_{\max }$ grid line is defincd to $b c$ the inlet to the cascade. This requircs equation (8) to be written in characteristic form only in the rrdirection:

$$
\begin{gathered}
\frac{\partial Q}{\partial t}+\frac{\partial \hat{F}}{\partial \xi}+C_{\xi} \\
+P\left(T^{-1} H T \frac{\partial U}{\partial \eta}+C_{\eta}\right)=0
\end{gathered}
$$

Consider the terms in the parentheses containing the information in the r-direction. Define:

$$
\left(T^{-1} H T \frac{\partial U}{\partial \eta}+C_{\eta}\right)=-\frac{\partial U}{\partial t_{\eta}}
$$

Multiply equation (14) by $T$ :

$$
T \frac{\partial U}{\partial t_{\eta}}+\mathrm{H} T \frac{\partial U}{\partial \eta}+T C_{\eta}=0
$$

The boundary conditions at the inlet boundary can be written in a form similar to equation (15):

$$
m_{k} \frac{\partial U}{\partial t_{\eta}}+\Pi_{k}+m_{k} C_{\eta}=0
$$

where

$$
I_{k}=\left\{\begin{array}{cc}
\mu_{k} m_{k} \frac{\partial U}{\partial \eta} & \text { for outgoing waves } \\
0 & \text { for incoming waves }
\end{array}\right.
$$

Here, $m_{k}$ are the left eigenvectors of $B, \mu_{k}$ are the cigenvalues of $B$, and $\frac{\partial U}{\partial \eta}$ is evaluated using one-sided differencing. The problem now becomes solving for $\frac{\partial U}{\partial t_{n}}$ from equation (16) and computing $\frac{\partial O}{\partial t}$ from equation (13):

$$
\frac{\partial O}{\partial t}+\frac{\partial F}{\partial \xi}+C_{\xi}=P \frac{\partial U}{\partial l_{n}}
$$

The boundary conditions applied at the inlet for subsonic inflow specify density, velocity and flow angle and solve equation (17) to determine the energy.

For viscous flows, the exit boundary conditions extrapolate density and velocity from the interior and specify static pressure to calculate energy. The characteristic equations are not valid across the viscous wake due to the non-isentropic flow at the exit boundary. This introduces reflective boundary conditions at the exit. It is not clear how to apply a non-reflective boundary condition in this region. For inviscid flows, the characteristic equations are used by specifying static pressure at the exit and solving an equation similar to equation (17) for density and the two components of velocity.

Solid wall boundary conditions are applied along the airfoil surface and the flow variables are averaged across the slit aft of the airfoil.

\section{Results and Discussion}

\section{NACA 65-Series Cascade}

Sample predictions were presented in reference 1 to validate the use of a deforming grid for an isolated airfoil. The present study investigates a NACA 65 -series cascade with subsonic inflow to help validate the solver for both zero and non-zero inter-blade phase angles. The predictions presented for this cascade are obtained from the solution of the full NavierStokes equations and therefore include viscous losses. The experimental data was obtained by Carta (ref. 11) and has been used by other researchers for code 
validation. This cascade has been called "The First Standard Configuration" by the Second International Symposium on Aeroelasticity in Turbomachines (ref. 12). Figure 2 shows the cascade geometry nomenclature used in the present investigation.

The cascade geometry consists of NACA 65-series thickness distributions on a 10 degrec circular-arc camber line, 55 degree stagger angle ( $\mathrm{v}$ ), a gap-tochord ratio $(\mathrm{g} / \mathrm{c})$ of 0.75 and a thickness-to-chord ratio ( $\mathrm{\tau}$ ) of 0.06 . An arbitrary test case from the First Standard Configuration has been chosen for comparison with the present solver. The inlet Mach number $\left(M_{1}\right)$ is 0.17 and the airfoils are pitching about the midchord with an amplitude $(\alpha)$ of 2.0 degrees and a reduced frequency $(k)$ of 0.123 based on scmi-chord. Two inter-blade phase angles (o) of 0 and 90 degrees are investigated in this study for comparisons with experimental data.

The grid used in the solution for this cascade is shown in Figure 3. The GRAPE code is used to gencrate a grid about the mean pitching angle. The solver is used to determine the mean flow solution. An iterative process is necessary by the user to verify that the specified exit pressure gives the desired velocity specified at the inlet. (Losses through the cascade prevent an exact analytical specification of these properties.) Four blades are required for $\sigma=90$ degrees, although only two are shown in Figure 3a. Multi-blade solutions for oscillating cascades are done by generating grids for each blade and using the mean flow solution as an initial condition to the unstcady solutions. The solver automatically gencrates these grids before the unsteady solution begins by deforming the mean flow grid for one blade through one cycle of oscillation and saving the grids that occur at multiples of the desired inter-blade phase angle. For example, when $\sigma=90$ degrees, four grids need to be assembled with a blade-to-blade phase angle of 90 degrees. The grid from the mean flow solution is used for the first blade ( $u t=0$ ). The grids for the remaining three blades are found by deforming the mean flow grid through one cycle of oscillation and storing the grid coordinates when $u t=90,180$, and 270 degrees. The solver numbers the blades and essentially stacks the grids to give one global grid containing four blades. The global grid is then used as the initial grid for the unsteady solution, where it is deformed for the oscillating cascade using the method described in the "Grid" section. Since the dynamic memory is usually limiting for multi-element grids, the solid-state storage device on the CRAYXMP at NASA Lewis Research Center is used to store all grids and flow information. The $\mathrm{C}$-grid and flow variables for one blade are transferred into dynamic memory on the CRAY as needed by the solver.

Figure $3 b$ shows more detail of the grid near the airfoil surface. The distance of the first grid line off the airfoil surface is 0.00005 chord lengths, which is appropriatc for modeling the boundary layer. The grid around one airfoil is $157 \times 40$ in the $\xi$ and r-directions, respectively. The grid is clustered about the leading edge and the boundary layer region. Also, the grid near the inlet is clustered in the r-direction to resolve waves near the inlet.

A steady-state solution was done first for the NACA 65-series cascade for the mean flow conditions. This required adjusting the exit pressure until the inlet Mach number was near 0.17. As mentioned by Verdon ( $\mathrm{ref} .13$ ), there is some ambiguity concerning the inlet flow angle. The experimental flow angle was $\beta_{i}=66.0$ degrees. Verdon found that $\beta_{1}=62.2$ degrees gave better agreement with the experimental mcan flow pressure distributions. A study of mean flow pressure distributions for various inlet flow angles is presented in Figure 4 using the present solver. An inflow angle of 64 degrees was found to give the best comparison with the experimental data. Each sólution required 2000 time steps to reach a steady-state and used about 400 seconds of CRAYXMP CPU time.

Two unstcady solutions for inter-blade phase angles of 0 and 90 degrees were done using the mean nlow solution as an initial condition. Both solutions were run with first-order temporal accuracy. The surface pressure time histories were recorded and found to reach a reasonably periodic solution after two cycles of cascade oscillation. A Fourier transform was done on the second cycle to determine the first harmonic magnitude and phase of the pressure distribution relative to the airfoil motion. Higher harmonics of the surface pressure distributions were found to be small compared to the fundamental frequency. The pressures are normalized by the airfoil pitching amplitude and the phase is referenced to the airfoil pitching angle starting at the maximum (nose up) blade angle. The predictions for $\sigma=90$ and 0 degrees are shown in Figure 5 along with the experimental data. Both the magnitude and phase are well predicted for both the upper and lower surfaces. 
The phase angles for $\sigma=0$ degrees differ quantitatively on the upper surface toward the trailing edge, although the overall trend is predicted. The reason for this is probably due to the pressure magnitudes in this region being close to zero. The unsteady calculations of phase are more sensitive when the output of the real and imaginary parts of pressure from the Fourier transform go towards zero. The unsteady solutions use $3.26 \times 10^{-5}$ seconds of CPU per time step per grid point per blade and required 16053 time steps to complete 2.25 cycles of oscillation.

\section{Elat Plate Cascade}

A cascade of flat plates was used in the present analysis for comparisons with the unsteady, smallperturbation, subsonic analysis in reference 16 . The cascade was arranged with $\gamma=53$ degrees and $g / c=0.767$, which corresponds to the conditions used in the biconvex airfoil cascade to be presented in the next section. All runs for the remaining solutions are inviscid so that the non-reflecting boundary conditions are valid at both the inlet and exit planes to the cascade. This also allows comparisons between runs using reflecting and non-reflecting boundary conditions.

The first set of flow conditions consider $M_{1}=0.65$, $\beta_{1}=53.0, k=0.221$, and $\alpha=0.10$ degrees. This gives a zero mean incidence angle on the flat plates and a small amplitude of oscillation to allow for comparisons with the small-perturbation, subsonic flat plate analysis of Smith (ref. 16). A $199 \times 33$ grid was used around a flat plate with $\tau=0.005$. The leading and trailing edges were rounded to aid the $\mathrm{C}$-grid generation, and therefore this is only an approximate representation of a flat plate. Figure 6 shows the comparisons for $\sigma=-90,90$, and 0 with the small-perturbation theory. (Notice that the pressure coefficient is now normalized by $\rho_{1} V_{1}^{2}$ instead of $\frac{1}{2} \rho_{1} V_{1}^{2}$ to be consistent with the normalization used by Smith in reference 16). Also shown in Figure 6 are comparisons from the present analysis for reflective versus non-reflective boundary conditions. The "reflective boundary conditions" means the inlet and exit planes specify the information from the mean flow solution. The "non-reflective boundary conditions" use the characteristic method described earlier to help minimize reflections from the inlet and exit.

For $\sigma=-90$ and $\sigma=90$, the agreement is very good for all cases and the type of boundary conditions had little effect on the results. When $\sigma=0$, the agreement with small-perturbation theory became worse and the type of boundary conditions used at the inlet and exit gave different the results within the code. In particular, the magnitude distribution dropped to values closer to the theory when using the non-reflective boundary conditions, but the phase values became worse. The reason for this is unknown, although this condition is very close to resonance, as calculated by Verdon (ref. 13). Acoustic resonance occurs in cascades when a wave propagates from an airfoil in the direction of the stagger line and intersects an adjacent blade in the same amount of time associated with the inter-blade phase angle. Numerical solutions are expected to be difficult when operating near an acoustic resonance condition.

Solutions were also done for oscillating flat plates with $M_{1}=0.80$ and $k=0.185$ and are compared to the small-perturbation theory. The results are presented in Figure 7 and show the same trends reported for Figure 6. The non-zero inter-blade phase angle solutions are in good agreement with the smallperturbation theory. The near-resonant condition, $\sigma=0$, shows poorer agreement with the theory. In general, the non-reflecting boundary conditions show good agreement in the magnitudes of the unsteady pressures when compared to the theory, but differ in the phase distributions. On the other hand, the cases using reflective boundary conditions show worse agreement in magnitude predictions and better agreement in phase distributions.

\section{NASA Lewis Cascade}

Another cascade geometry for which experimental data (including unsteady surface pressure distributions) exists is the NASA Lewis Transonic Oscillating Cascade (ref.14 and ref. 15). This cascade consists of symmetric biconvex airfoils $\left(\tau^{\prime}=0.076\right)$ arranged with $\gamma=53$ degrees and $g / c=0.767$. Both subsonic and transonic test cases have been chosen from the experimental data for comparisons with predictions from the present solver. Again, all runs for the remaining solutions are inviscid so that a boundary condition study can be performed.

The subsonic test cases consider $M_{1}=0.65$ and the mean $\beta_{1}=60.0$ degrees ( $i=7$ degrees). The grid generation is similar to the technique described for the NACA 65-series cascade. The distance of the first 
grid line off the airfoil surface is 0.01 chord lengths. The grid around one airfoil is $199 \times 22$ in the $\xi$ and n-directions, respectivcly. Unsteady solutions were done for an oscillating cascade with the biconvex airfoils, $\alpha=1.2$ degrees, $k=0.221$, and $\sigma=-90,90$ and 0 and are shown in Figures 8,9 , and 10, respectively. A Fourier transform was done on the third cycle to determine the first harmonic unsteady pressure distributions. For completeness, both the $\mathrm{CP}$ and $\triangle C P$ predictions are presented for real and imaginary parts, and magnitude and phase components. The cases for $\sigma=-90$ (Figure 8 ) and $\sigma=9)$ (Figure 9) are in good overall agreement with the experimental data of reference 15 . When $\sigma=0$ (Figure 10), there is a shift in the real part (in-phase part) of the unsteady pressure on both the upper and lower surfaces relative to the experimental data. Again, operating near an acoustic resonant condition may contribute to these discrepencies. It is interesting to note that while the upper and lower unsteady surface pressures are in poor agreement with the data, the $\triangle C P$ representation of the same predictions look better. This illustrates the importance of presenting separate pressure distributions for the upper and lower surfaces when possible.

An investigation of the numerical timc accuracy is done for the same test cases presented above. The code can be run with either first-order or secondorder accuracy in time. Figure 11 compares the real and imaginary parts of pressure for $\sigma=-90,9)$, and 0 , and shows that there are only minor differences between the predictions for first and second-order temporal accuracy.

The transonic test cases are identical to the subsonic test cases, except $M_{1}=0.80$. The increase in the inlet velocity causes a shock to form on the upper surface near the leading edge. Figure 12 shows the mean flow pressure distributions for two conditions: 1.) matching the experimental inlet Mach number $(0.80)$ and 2.) matching the experimental ratio of the exit static pressure to the inlet total pressure (0.7248). The desired flow conditions probably lic somewhere between these predictions, as shown by the comparison with experimental data. In both cases, the pressure distributions are in good agrecment with each other and the experimental data and therefore either condition could be used for the mean flow in the unsteady analysis. The unsteady solutions (that follow) match the inlet flow velocily from the experimental data.
The unstcady pressure distributions for $\mathrm{k}=0.185$, $\sigma=-90,90$, and 0 degrees are shown in Figures 13, 14, and 15 , respectively. The change in the reduced frequency maintains the same oscillation frequency used in the subsonic cases ( $\omega=200 \mathrm{~Hz}$ ). The agreement with cxperimental data are very good for the $\sigma=-90$ degrees case (Figure 13). The shock on the upper surface is modeled by the analysis, as evident by the pressure peaks near the leading edge. Note that the experiment did not have pressure transducers near this region and is not expected to capture this behavior. The agrecment with the data becomes progressively worse for $\sigma=90$ (Figure 14) and $\sigma=0$ (Figure 15). The real-part of pressure on the forward portion of the upper surface differs from the data for $\sigma=90$. This was also observed by Verdon (ref. 13) using a linearized potential code. Both the real and imaginary parts of pressure differ from experiment for $\sigma=0$. Again, this case is near an acoustic resonance condition. Also, the real and imaginary parts of pressure are significantly smaller than other cases, which makes the calculations for phase sensitive to their values.

A study was done to determine the effect of boundary conditions on the first harmonic pressure distributions. Each of the three cases reported above were run with four combinations of inlet and exit houndary condition types: 1.) reflecting inlet and exit, 2.) "non-reflecting" inlet and exit, 3 .) reflecting inlet, "non-reflecting" cxit, and 4.) "non-reflecting" inlet, reflecting exit. The results are presented in Figure 16 and show that the type of boundary conditions can make a significant difference on the first harmonic unsteady pressure distributions. For clarity, the individual cases are labeled only when there are noticable differences in the pressure distributions. The type of boundary condition used has the little effect when $\sigma=-90$ degrees. However, when $\sigma=90$ degrees, the inlet boundary condition has a significant effect, particularly near the leading edge shock. The zero interblade phase angle case, which is near an acoustic resonance condition, shows a different solution for each type of boundary condition. This is not to say that these observations will hold true for other flow conditions. Much work has been done by other researchers to predict when acoustic waves will propagate from an oscillating blade row. They have shown that different geometries and flow conditions will change the propagation characteristics. While a full study of wave propagation is beyond the scope of 
this work, the present study does show that proper treatment of the inlet and exit boundary conditions is neccessary for modeling the first harmonic unsteady pressure distributions in transonic flows. Overall, the results from the boundary condition studies show that the type of inlet boundary conditions have a greater effect on the unsteady pressure distributions than the type of exit boundary conditions. This is encouraging for validating the viscous solutions that cannot use the non-reflective boundary conditions at the exit. However, this conclusion is not substantiated without a further study on the effects of inlet and exit distance from the blade surface. All of the present cases have an inlet boundary closer to the blade than the exit boundary. Therefore, the downstream-running waves have a longer distance for altenuation than the upstream-running waves.

The inviscid runs require about $2.35 \times 10^{5}$ seconds of $\mathrm{CPU}$ per time step per grid point per blade. The number of time steps required for one cycle of oscillation is a function of $\mathrm{k}, M_{1}$, and $\wedge \mathrm{t}$. The cases with $\mathrm{k}=0.185, M_{1}=0.80$ and $\Delta \mathrm{\tau}=0.01$ require 2835 seconds of CPU for 6899 iterations, which corresponds to 3.25 cycles of oscillation of four blades. It is possible to reduce this run time by performing a grid and time step size study.

\section{Conclusions}

A compressible, Euler or full Navier-Stokes, finite difference code has been developed for modeling inviscid and viscous flow through oscillating cascades. The code uses a deforming grid technique to capturc the motion of the airfoils and can model oscillating cascades with both zero and non-zero inter-blade phase angles. Two-dimensional, unsteady characteristic boundary conditions are applied at the inlet for viscous solutions and to both the inlet and exit for inviscid solutions to minimize wave reflections from these regions. Results show that predictions for a low speed, NACA 65-series oscillating cascade are in good agreement with experimental data. The predictions for an oscillating cascade of flat plates are in good agreement with small-perturbation theory for non-zero inter-blade phase angles. The zero degree inter-blade phase angle cases, which were near an acoustic resonant condition, differ from the theory. The predictions for an oscillating cascade of biconvex airfoils are in fair agreement with experimental data for non-zero inter-blade phase angles. Solutions for zero degree inter-blade phase angles only show qualitative agreement with the data. Studies on reflecting versus non-reflecting inlet and exit boundary conditions show that the treatment of the boundary can have a significant effect on the first harmonic, unsteady pressure distributions for the cases with zero inter-blade phase angles. Using first or secondorder temporal accuracy in the numerical algorithm did not make a significant difference in the unsteady pressure distributions for the present solutions.

\section{Acknowledgements}

The author wishes to thank Dr. L.N. Sankar of the (icorgia Institute of Technology, and Dr. Danicl lloyniak and Danicl Buffum of the NASA Lewis Research Center for their many helpful discussions on this work.

\section{References}

1. Huff, D.L., "Numerical Simulations of Unsteady, Viscous, Transonic Flow Over Isolated and Cascaded Airfoils Using a Deforming Grid," AIAA Paper 87-1316, Junc 1987.

2. Sankar, N.L. and Tang, W., "Numerical Solution of Unsteady Viscous Flow Past Rotor Sections," AIAA Paper 85-0129, Jan. 1985.

3. Baldwin, B. and Lomax, H., "Thin-Layer Approximation and Algebraic Model For Separated Turbulent Flows,"AIAA Paper 78-257, Jan. 1978.

4. Johnson, D.A. and King, L.S., "A New Turbulence Closure Model for Boundary Layer Flows with Strong Adverse Pressure Gradients and Seperation," AIAA Paper 84-0175, Jan. 1984.

5. Gorski, J.J., "A New Near-Wall Formulation for the $\mathrm{K}+$ Equations of Turbulence," AIAA Paper 86-0556, Jan. 1986.

6. Wu, J.C., "A Numerical Study of Turbulent Flow Past Airfoils," Ph.D. Thesis, Georgia Institute of Technology, 1988.

7. Sorenson, R.L., "A Computer Program to Generate Two-Dimensional Grids About Airfoils and Other Shapes by the Use of the Poisson's Equations," NASA TM-81198, 1980.

8. Chima, R.V., "Explicit Multi-Grid Algorithm for Quasi Three-Dimensional Viscous Flow in Turbomachinery," Journal of Propulsion and Power, Vol.3, No.5, Sept.-Oct. 1987, pp. 397-405. 
9. Hedstrom, G.W., "Nonreflecting Boundary Conditions for Nonlinear Hyperbolic Systems," Journal of Computational Physics, Vol.30, 1979, pp.222-237.

10. Thompson, K.W., "Lecture Series in Computational Fluid Dynamics," NASA TM-100010, Aug. 1987.

11. Carta, F.O., "An Experimental Investigation of Gapwise Periodicity and Unsteady Aerodynamic Response in an Oscillating Cascade," Vol. I Experimental and Theoretical Results, NASA CR-3513, Dec. 1981.

12. Suter, P. (editor), Aeroelasticity in Turbomachines, Proceedings of the Second International Symposium held in Lausanne, Switzerland, Sept. 8-12, 1980, Juris-Verlag, Zurich, 1981.

13. Verdon, J.M., "Application of a Linearized Unsteady Aerodynamic Analysis to Standard Cascade Configurations," NASA CR-3940, Jan.
14. Shaw, L.M., Boldman, D.R., Buggele, A.E., and Buffum, D.H., "Unsteady Pressure Measurements on a Biconvex Airfoil in a Transonic Oscillating Cascade," ASME Journal of Engineering for Gas Turbines and Power, Vol.108, No.1, Jan. 1985.

15. Buffum. D.H., Boldman, D.R., and Fleeter, S., "The Unsteady Aerodynamics of an Oscillating Cascade in a Compressible Flow Field," NASA TM-100219, Sept. 1987.

16. Smith, S.N., "Discrete Frequency Sound Generation in Axial Flow Turbomachines," ARC-R/M3709, 1971.

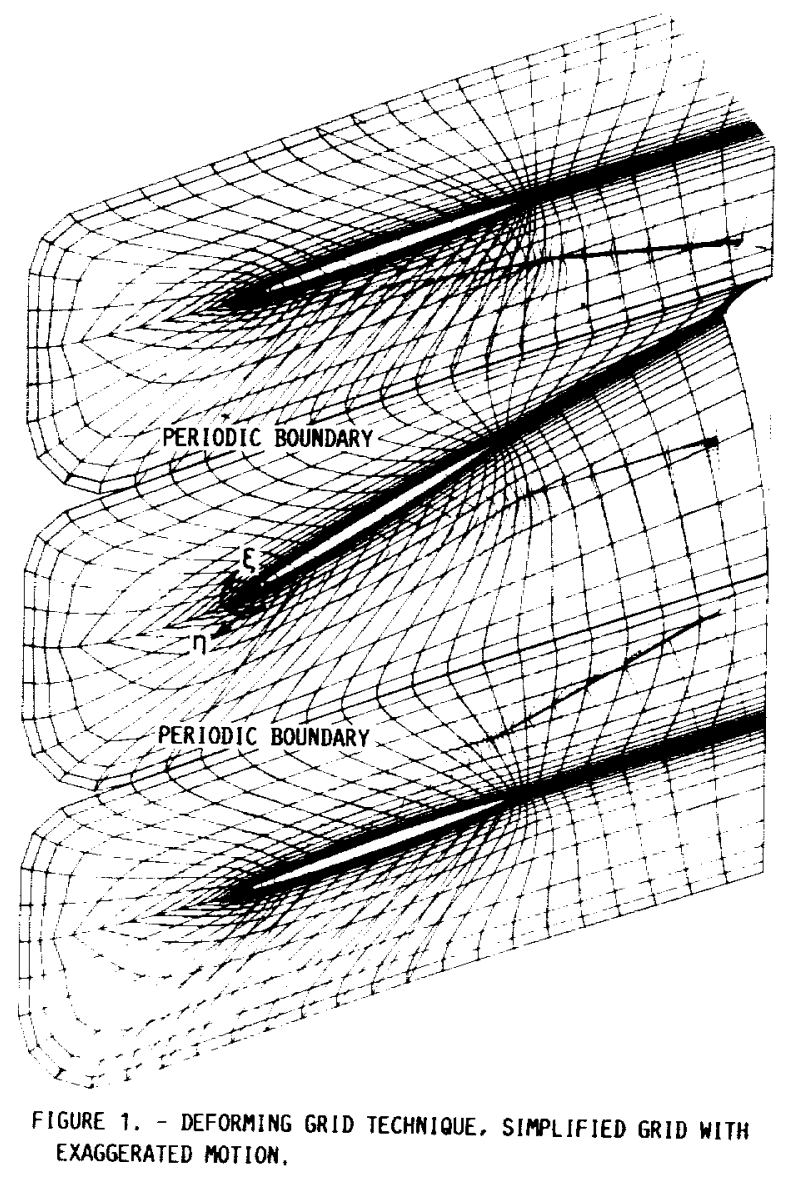




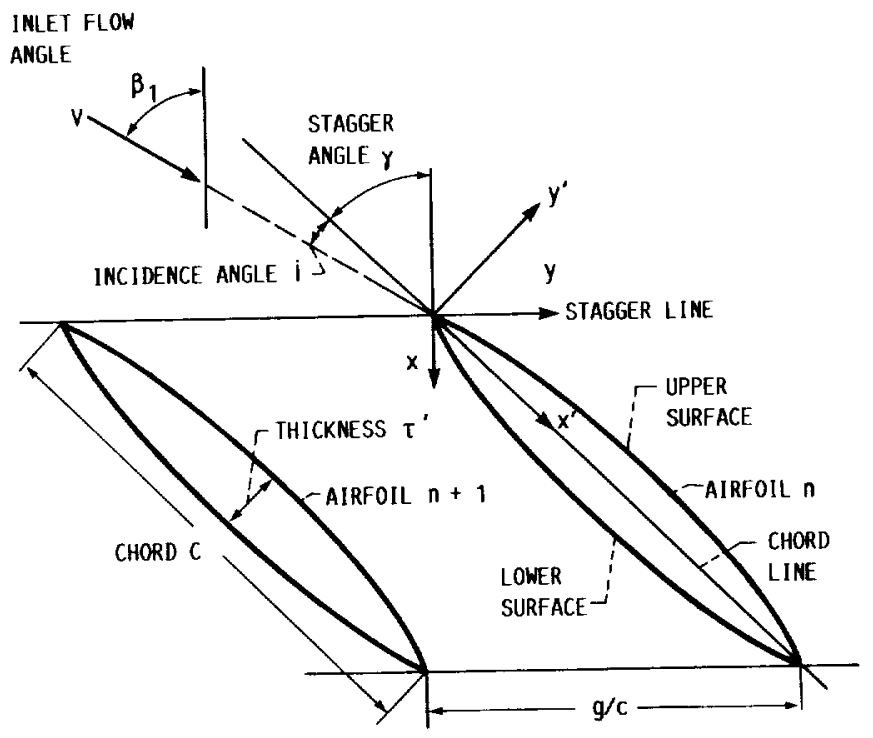

FIGURE 2. - CASCADE GEOMETRY.

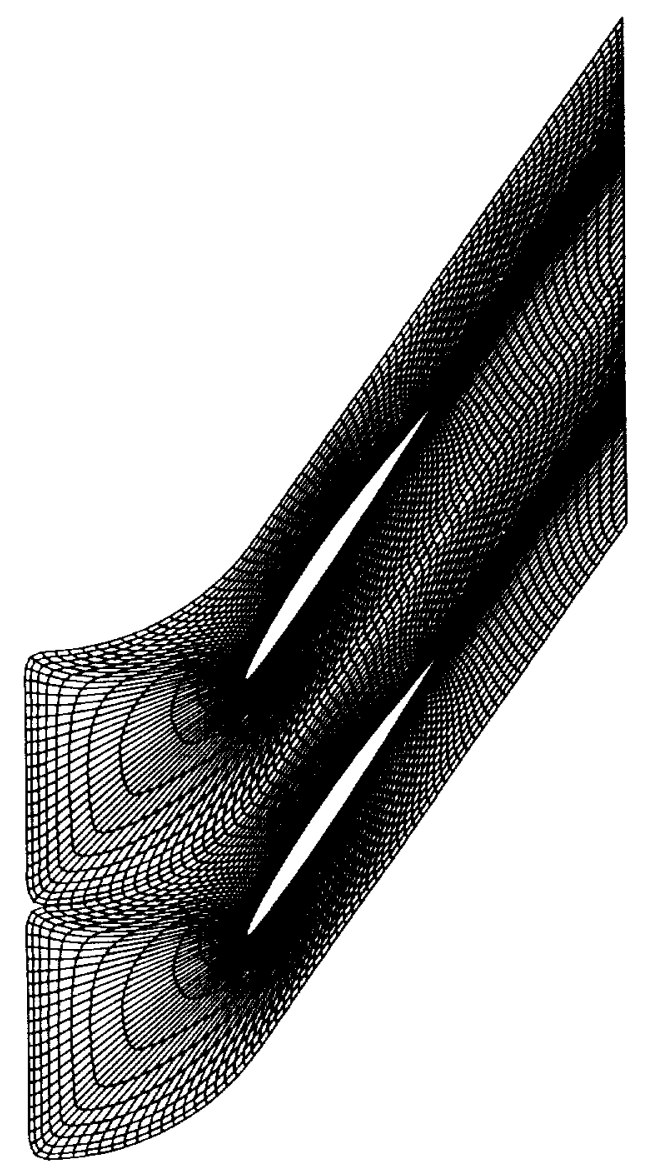

(A) STEADY-STATE GRID FOR IHE NACA 65-SERIES CASCADE.

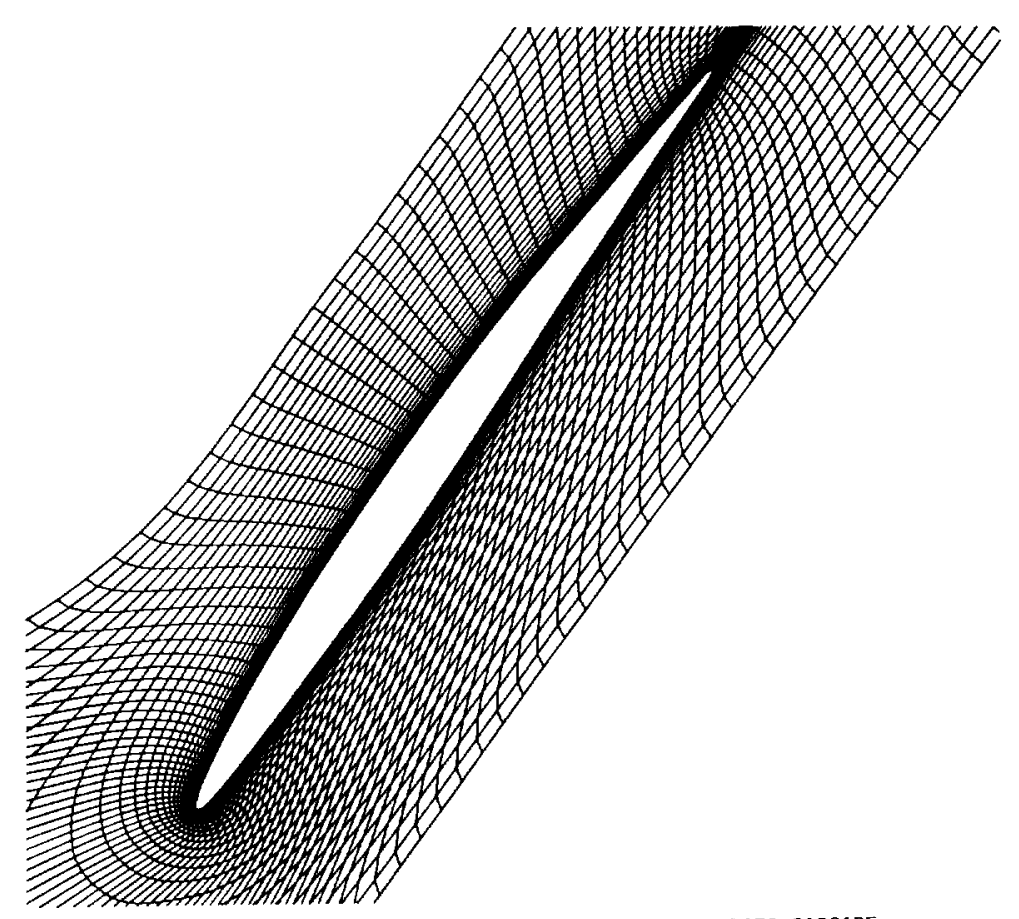

(B) GRID DEIAIL NEAR AIRFOIL FOR THE NACA 65-SERIES CASCADE.

FIGURE 3. 


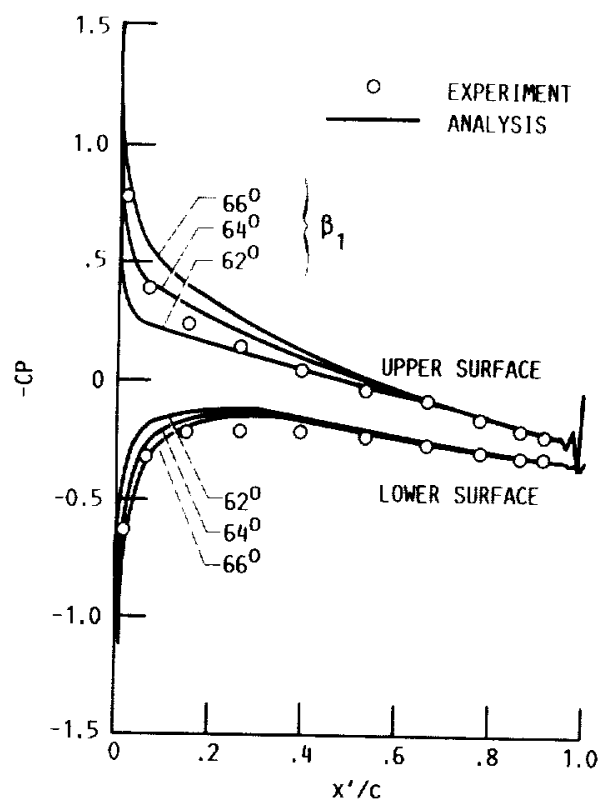

FIGURE 4. - MEAN FLOW PRESSURE DISTRIBUTION FOR NACA 65-SERIES CASCADE, $M_{1}=0.17, Y=55^{\circ}, g / C=0.75$.

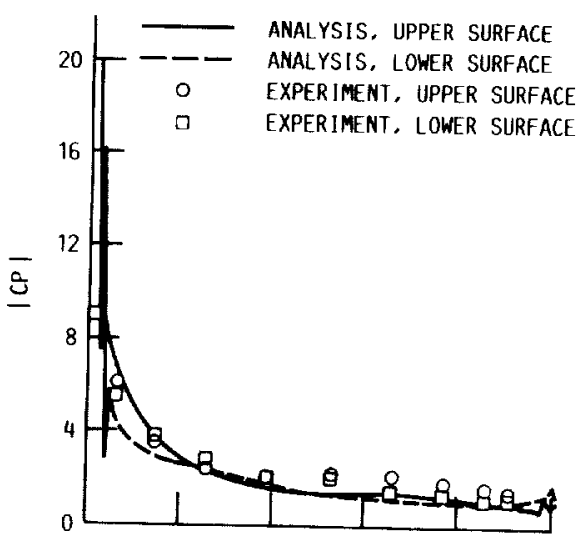
(A) $0=90^{-180}$.
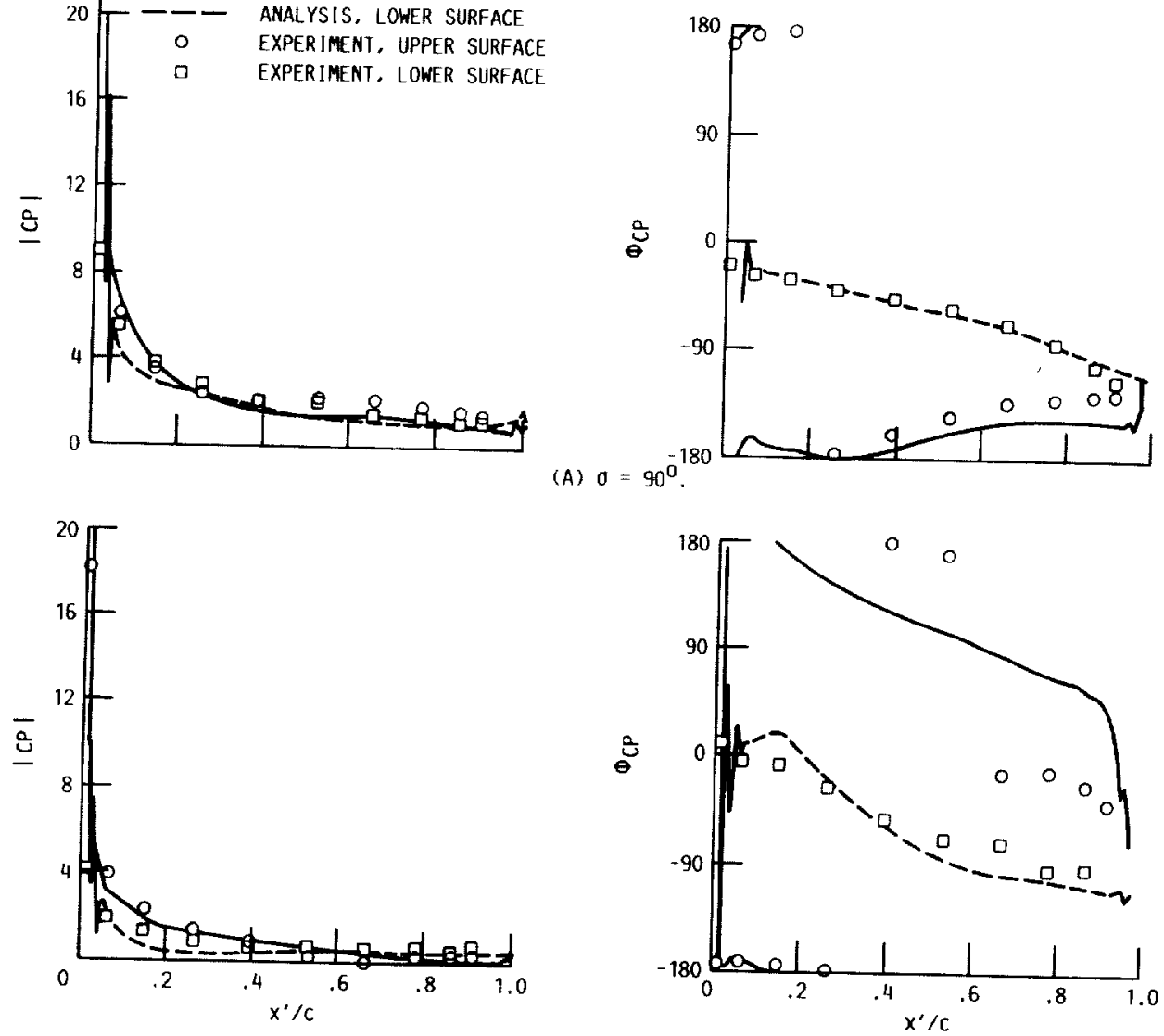

(B) $\sigma=0^{\circ}$.

FIGURE 5. - FIRST HARMONIC PRESSURE DISTRIBUTION FOR NACA 65-SERIES OSCILLATING CASCADE, $M_{1}=0.17, \beta_{1}=64^{\circ} \pm 2^{\circ}, k=0.123$. 

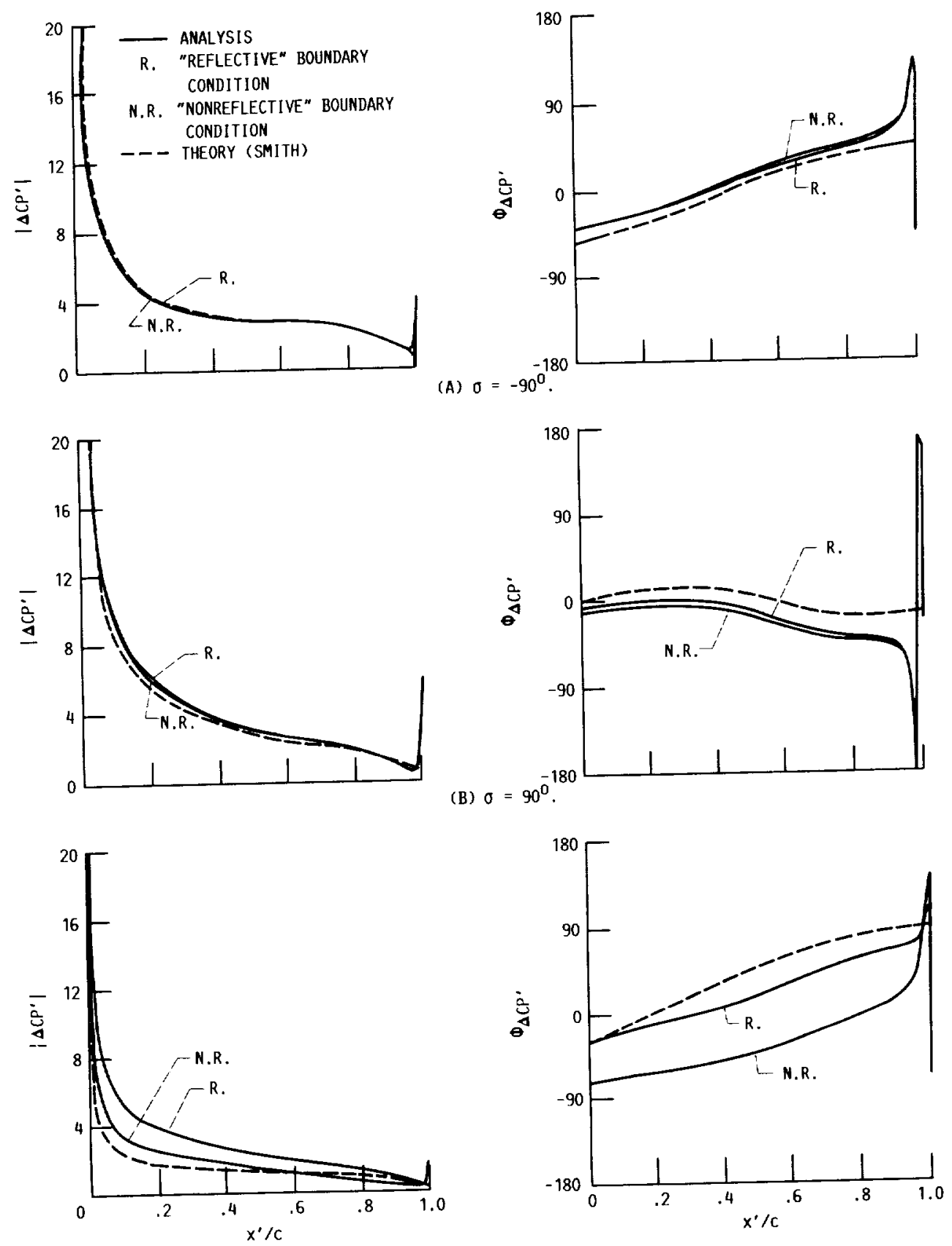

(C) $\sigma=0^{0}$.

FIGURE 6. - BOUNDARY CONDITION AND THEORY COMPARISON FOR FLAT PLATE CASCADE, $M_{1}=0.65$. $\beta_{1}=53^{\circ} \pm 0.10^{\circ}, k=0.221$. 

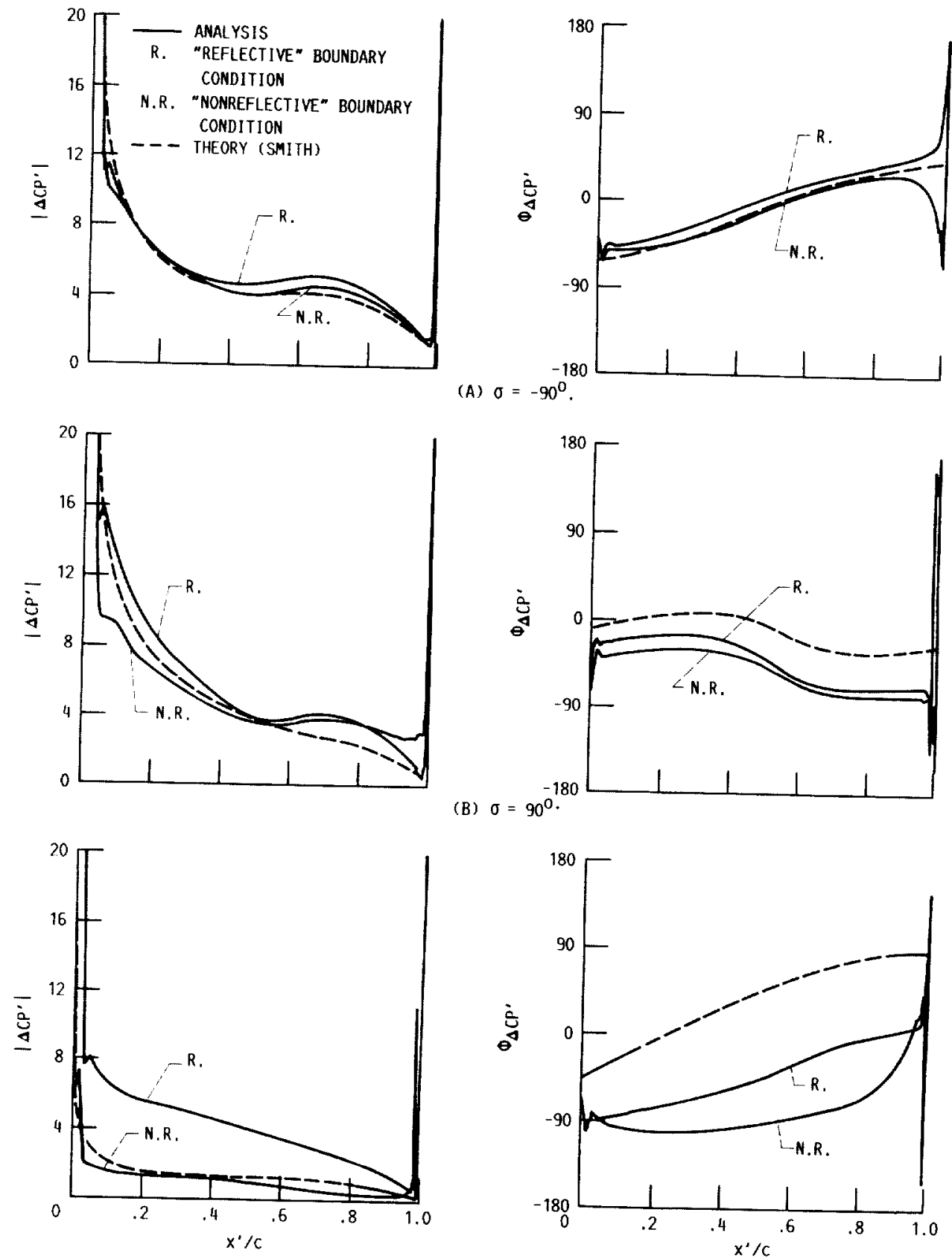

(C) $a=0^{0}$. FIGURE $7,-$ BOUNDARY CONDITION AND THEORY COMPARISON FOR FLAT PLATE CASCADE, $M_{1}=0.80$.
$\beta_{1}=53^{\circ} \pm 0.10^{\circ}, k=0.185$. 

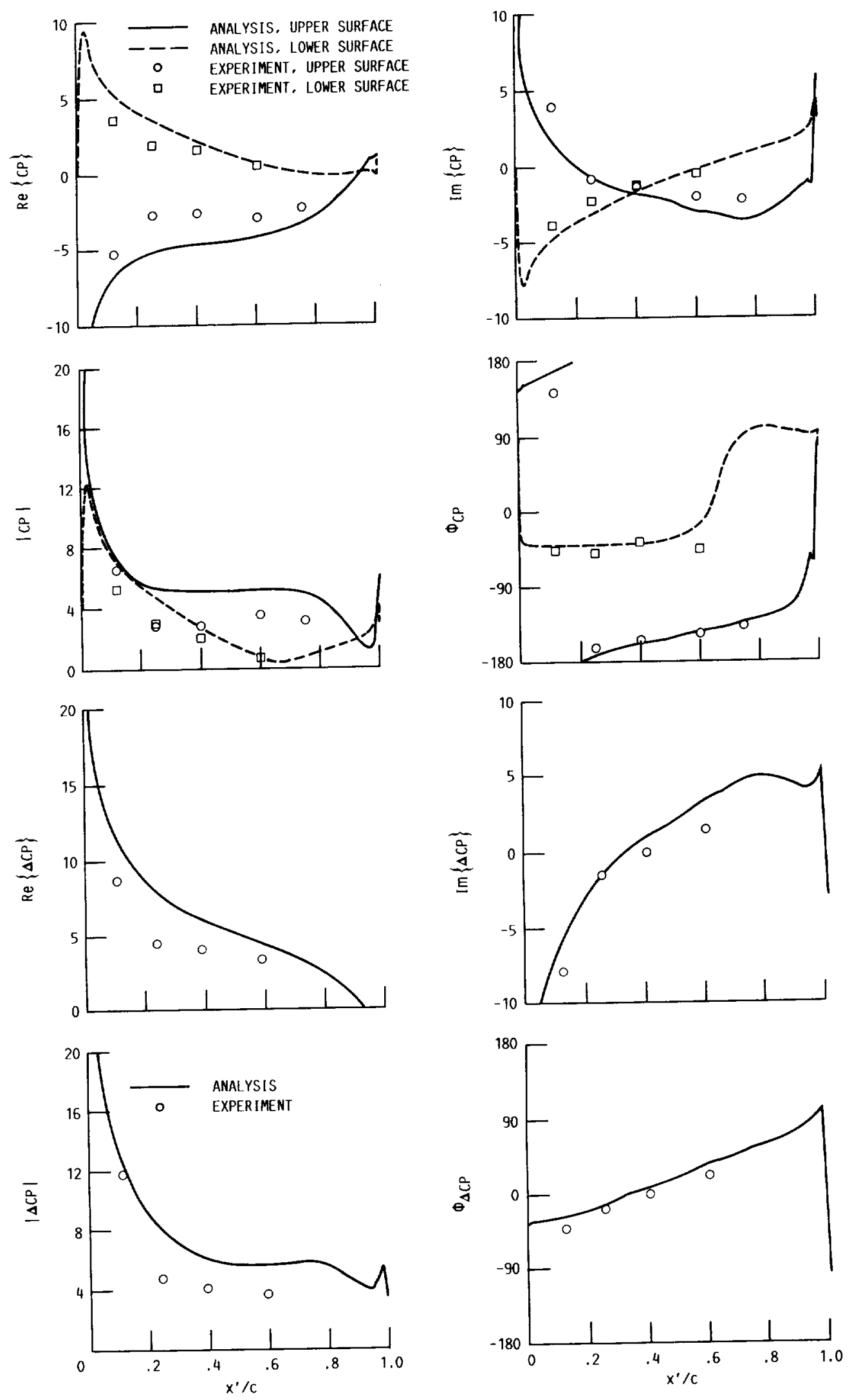

FIGURE 8. - FIRST HARMONIC PRESSURE DISTRIBUTION FOR BICONVEX AIRFOIL CASCADE, $m_{1}=0.65$.

$\beta_{1}=60^{\circ} \pm 1.2^{\circ}, k=0.221, \sigma=90^{\circ}$. 

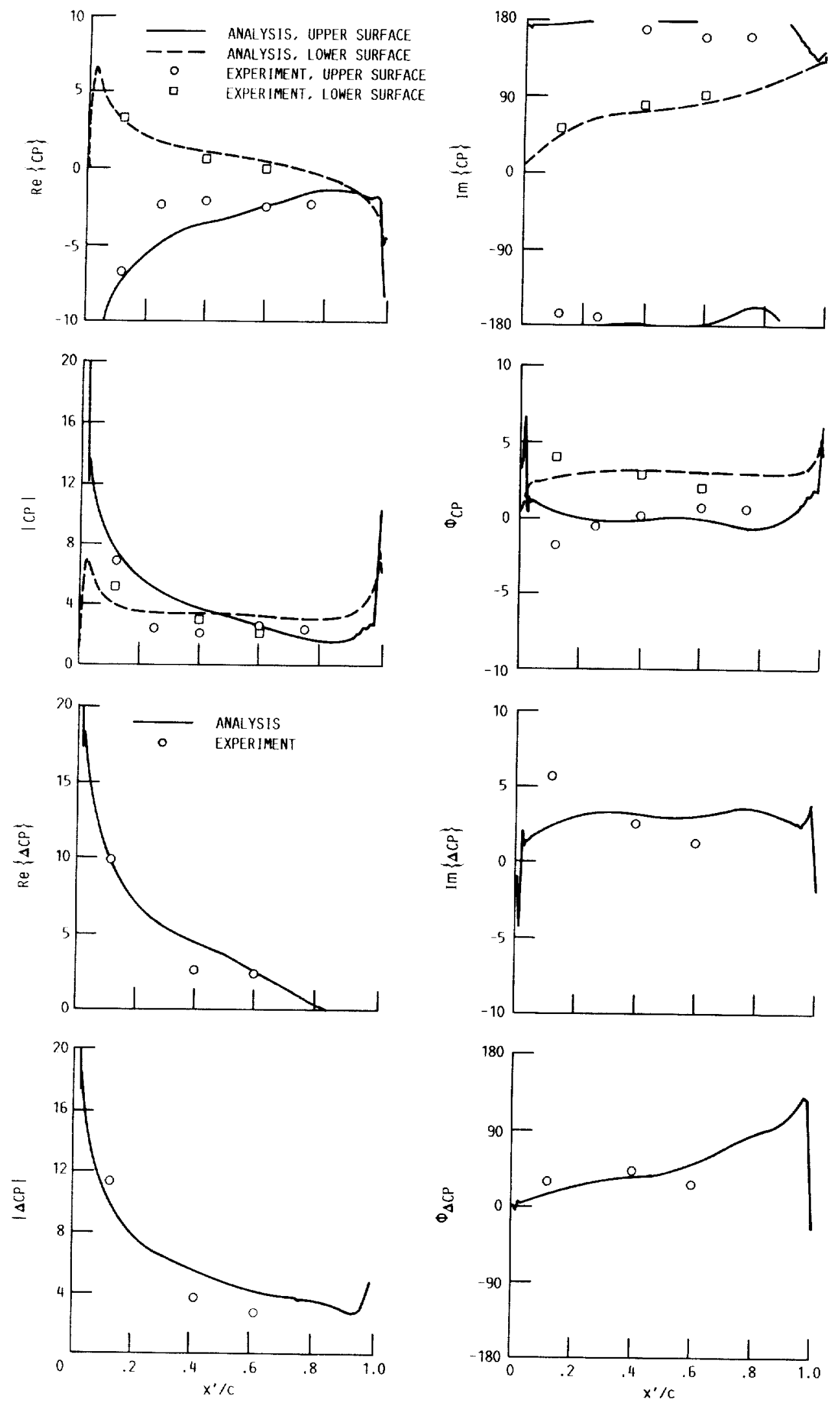

FIGURE 9. - FIRST HARMONIC PRESSURE DISTRIBUTION FOR BICONVEX AIRFOIL CASCADE, $M_{1}=0.65$ $\beta_{1}=60^{\circ} \pm 1.2^{\circ}, k=0.221, \sigma=90^{\circ}$. 

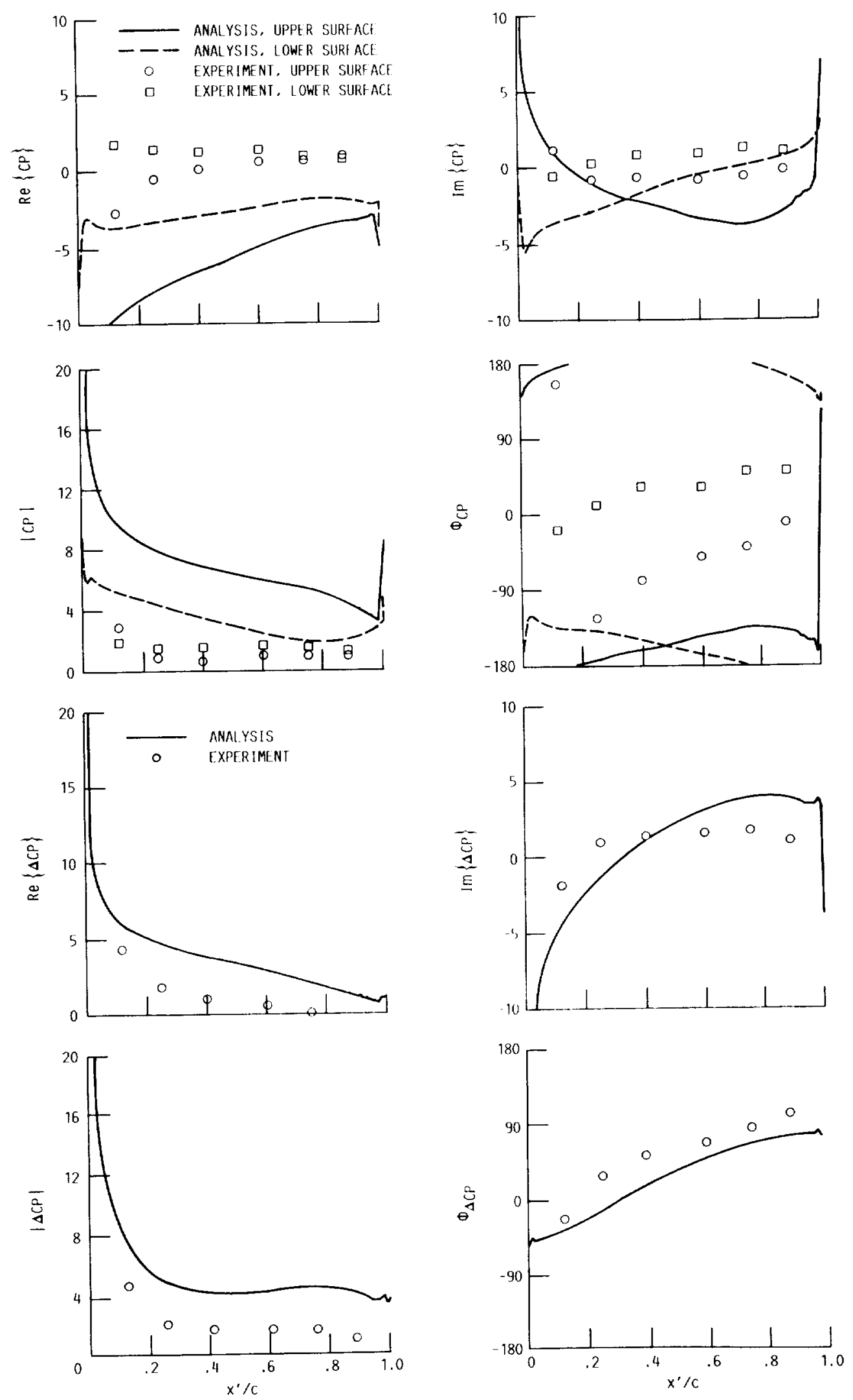

FIGURE 10. - FIRSI HARMONIC PRESSURF DISTRIBUTION FOR BICONVEX AIRFOIL CASCADE, $M_{1}=0.65$, $\beta_{1}=60^{\circ} \pm 1.2^{\circ}, k=0.221, \sigma=0^{0}$. 

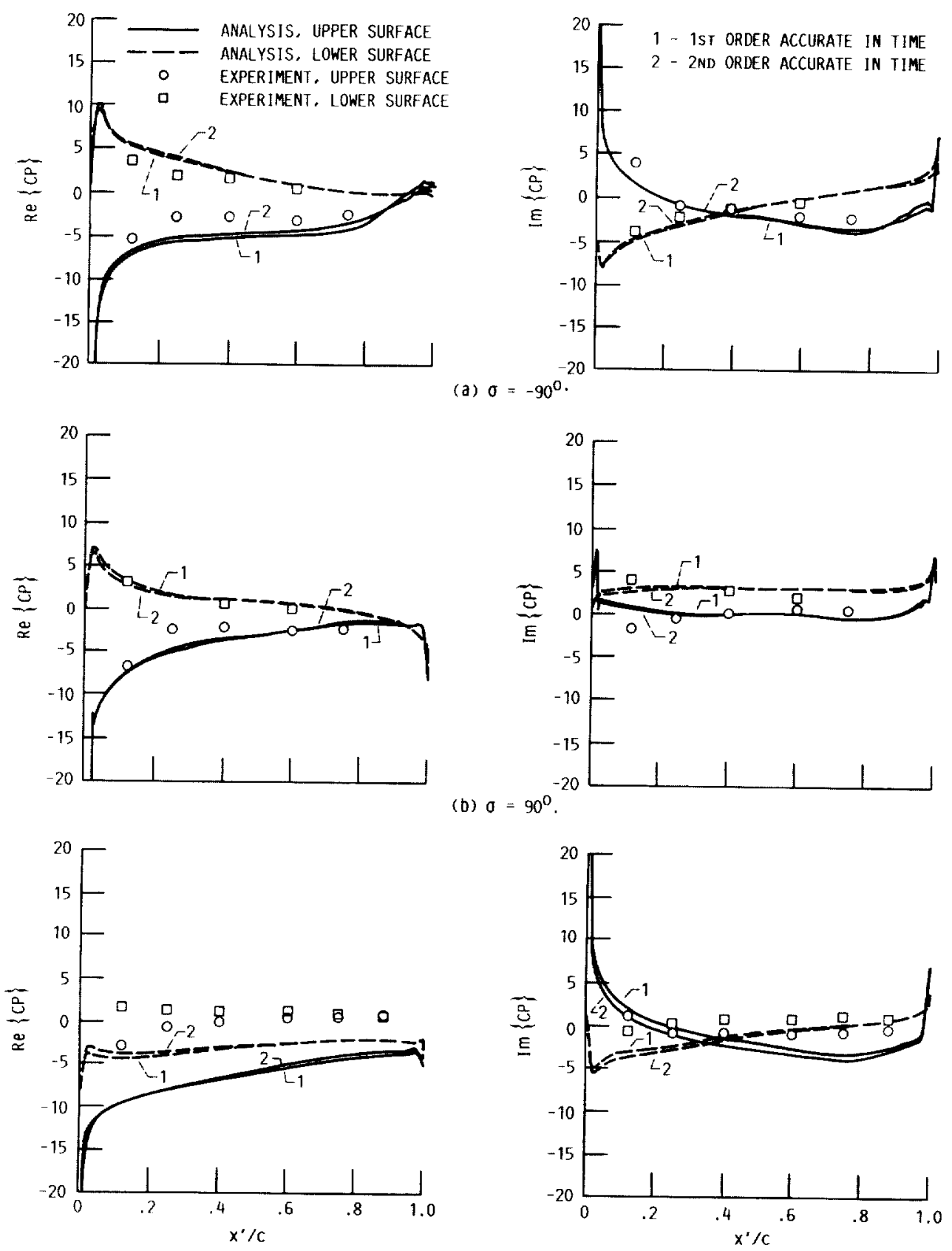

(c) $\sigma=0^{\circ}$.

FIGURE 11. - TIME ACCURACY COMPARISON FOR FIRST HARMONIC PRESSURE DISTRIBUTION FOR BICONYEX AIRFOIL CASCADE, $m_{1}=0.65, \beta_{1}=60^{\circ} \pm 1.2^{\circ}, k=0.221$. 


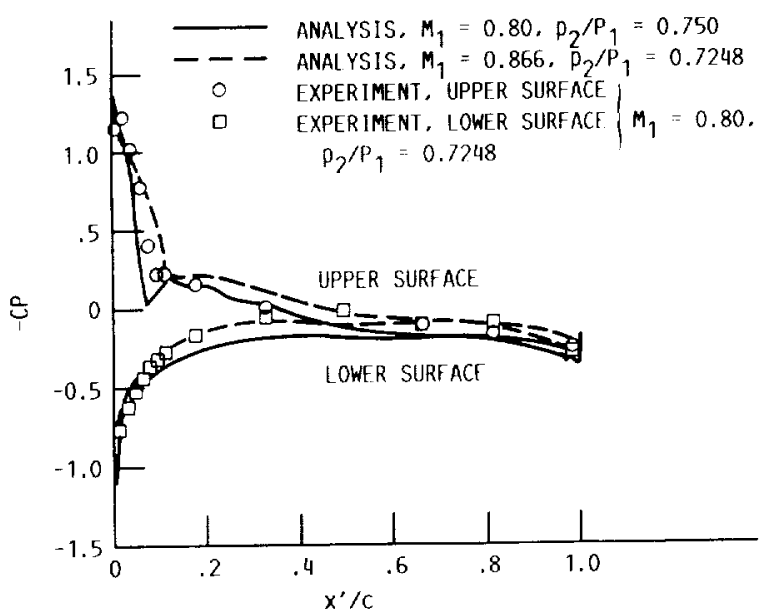

FIGURE 12, - MEAN FLOW PRESSURE DISTRIBUTION FOR BICONYEX AIRFOIL CASCADE, $\beta_{1}=60^{\circ}, \gamma=53^{\circ}, g / c=$ $0.767, T^{\prime}=0.07$, 

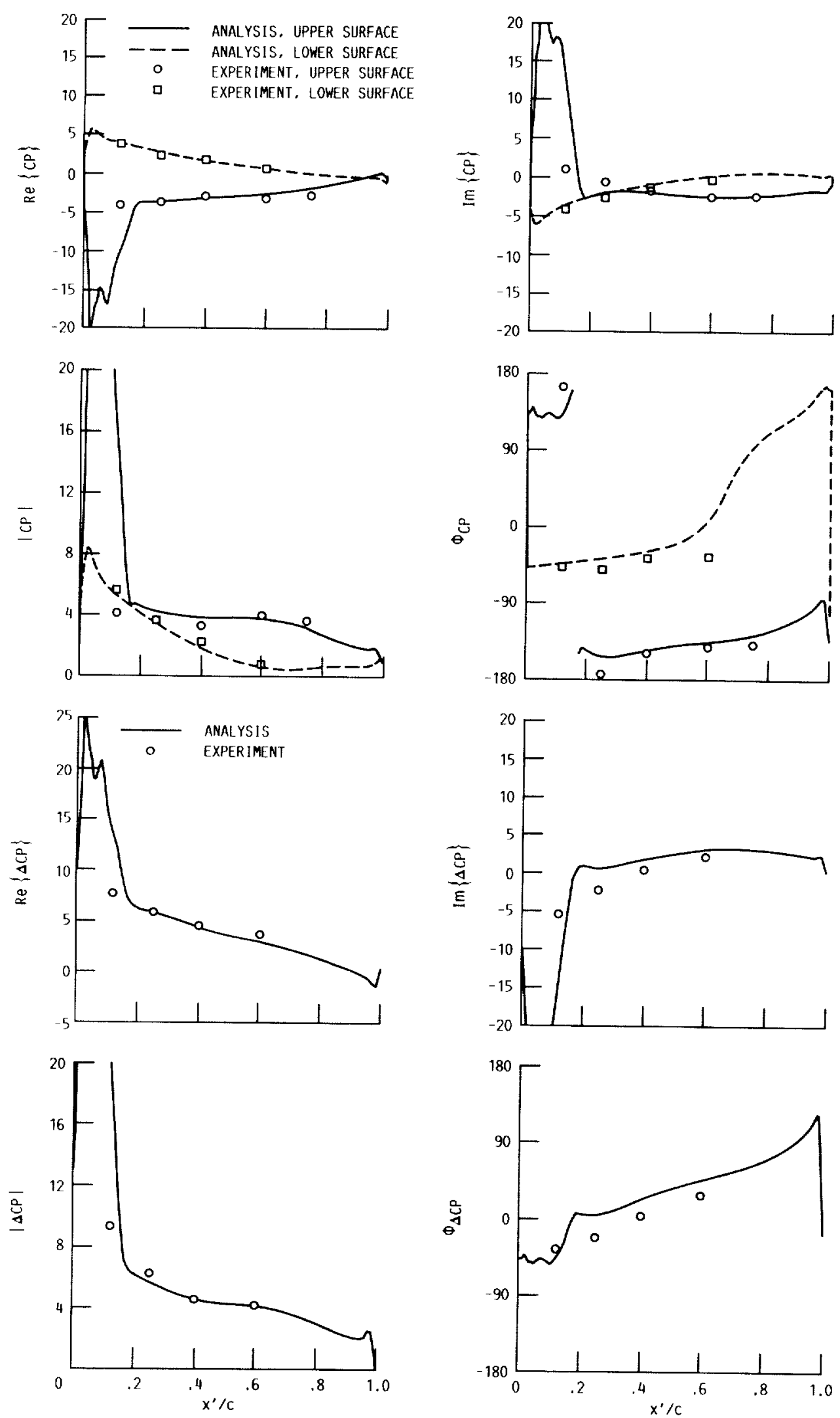

FIGURE 13. - FIRST HARMONIC PRESSURE DISTRIBUTION FOR BICONVEX AIRFOIL CASCADE, $m_{1}=0.80$, $\beta_{1}=60^{\circ} \pm 1.2^{\circ}, k=0.185, \sigma=-90^{\circ}$. 

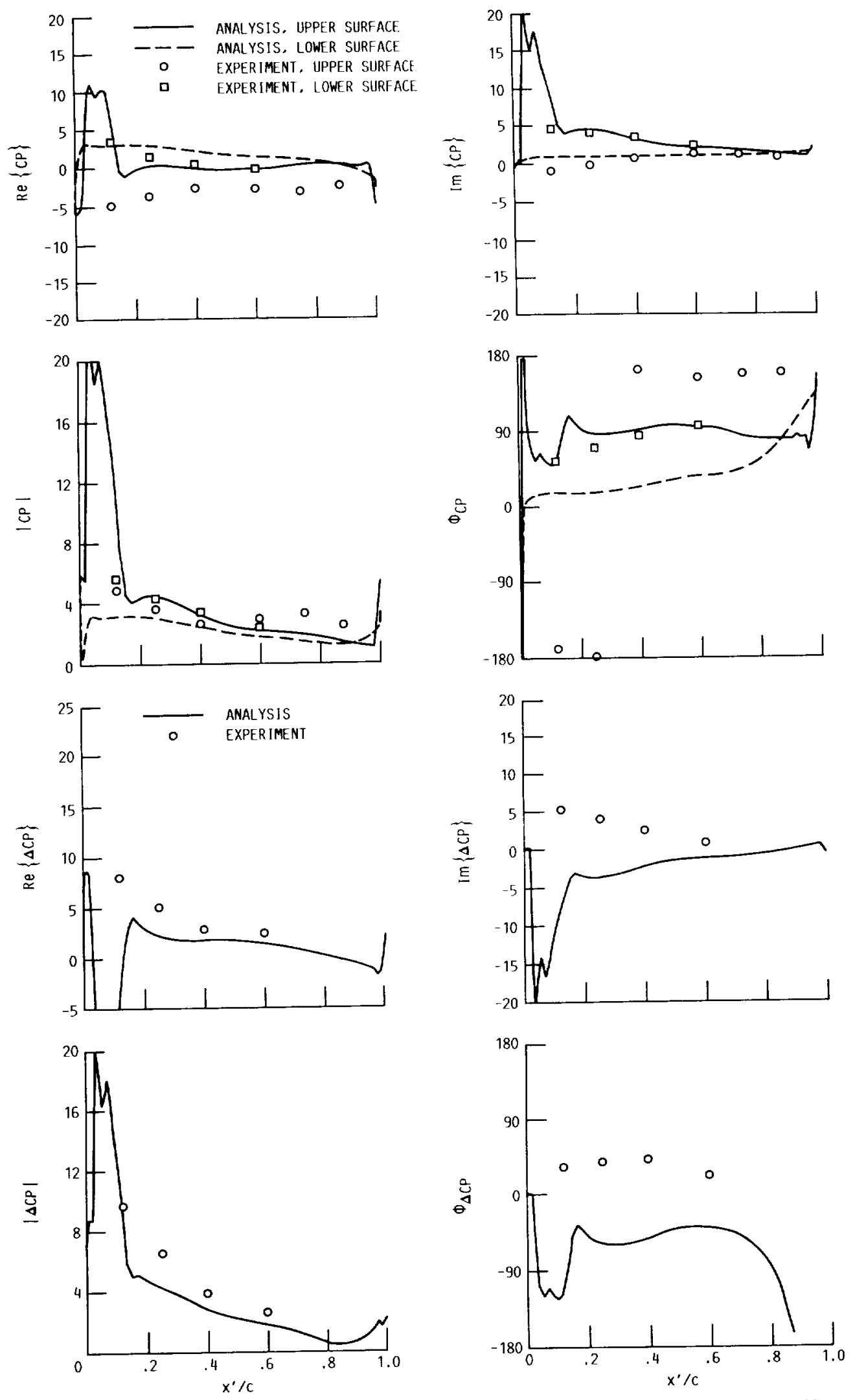

FIGIIRE 14 . FIRST HARMONIC PRESSURE DISTRIBUTION FOR BICONYEX AIRFOIL CASCADE, $m_{1}=0.80$. $\beta_{1}=60^{\circ} \pm 1.2^{\circ}, k=0.185, \sigma=90^{\circ}$. 

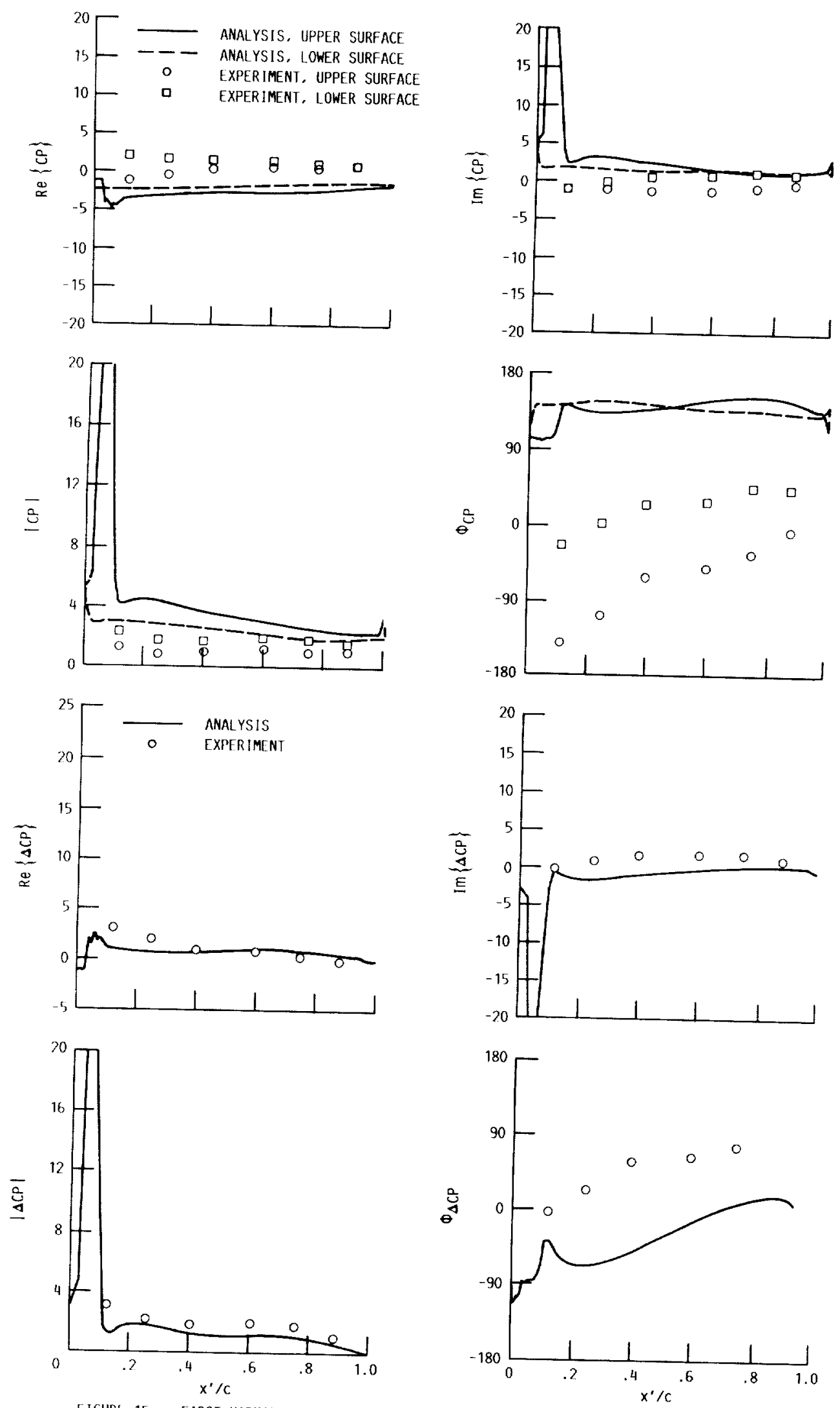

FIGURE 15. - FIRST HARMONIC PRESSURE DISTRIBUIION FOR BICONVEX AIRFOIL CASCADE, $M_{1}=0.80$,

$\beta_{1}=60^{\circ} \pm 1.2^{\circ}, k=0.185, \sigma=0^{\circ}$. 

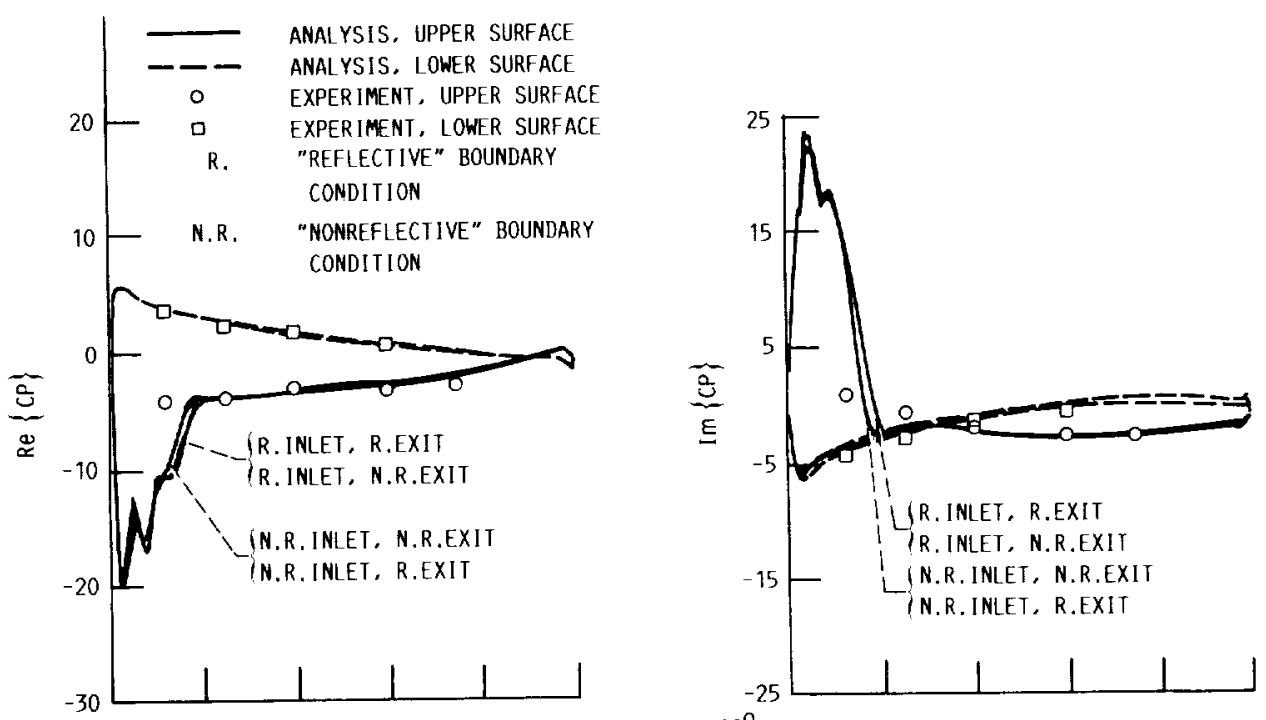

(a) $\sigma=-90^{\circ}$.
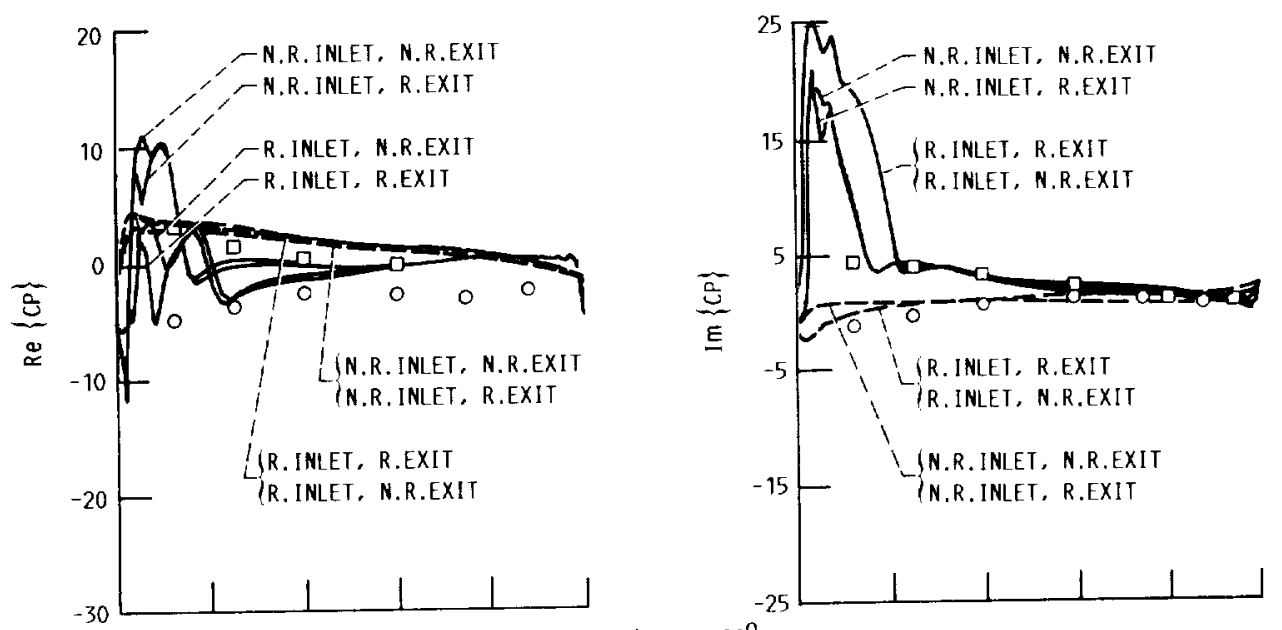

(b) $0=90^{\circ}$.
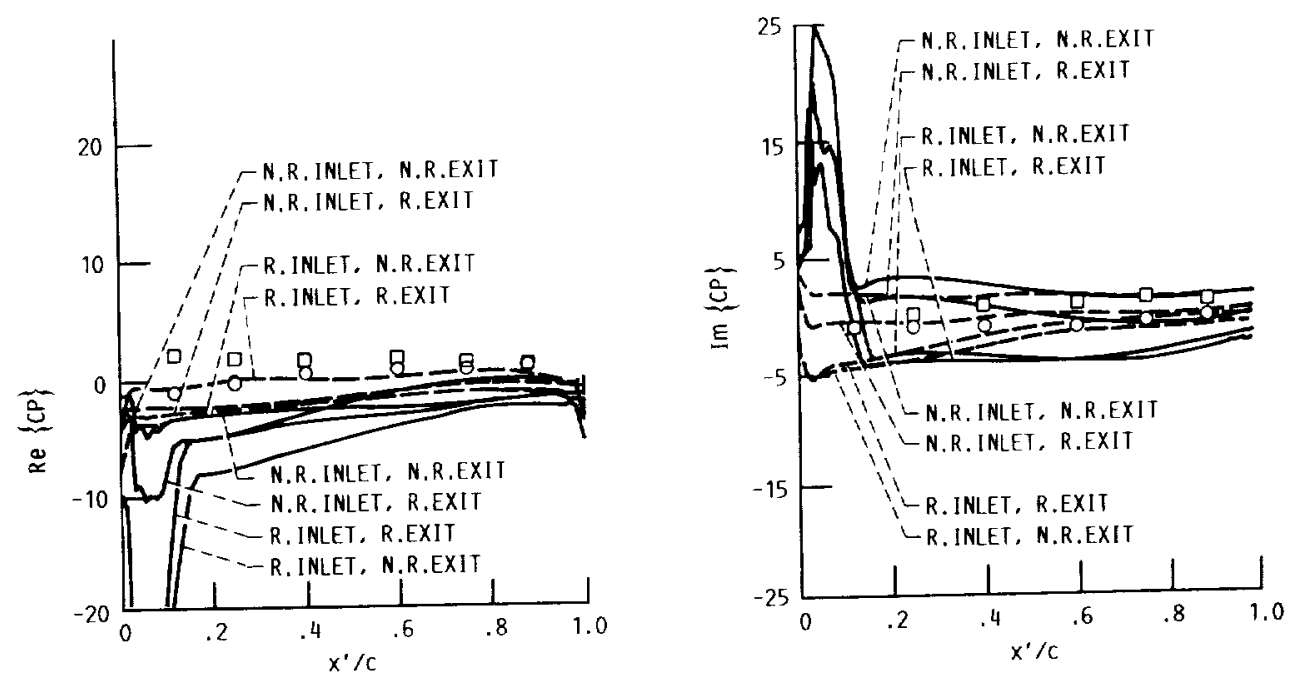

(c) $\sigma=0^{0}$.

FIGURE 16 - BOUNDARY CONDITION STUDY FOR FIRST HARMONIC PRESSURE DISTRIBUTION FOR BICONVEX AIRFOIL CASCADE. $M_{1}=0.80, \beta_{1}=60^{\circ} \pm 1.2^{\circ}, k=0.185$. 


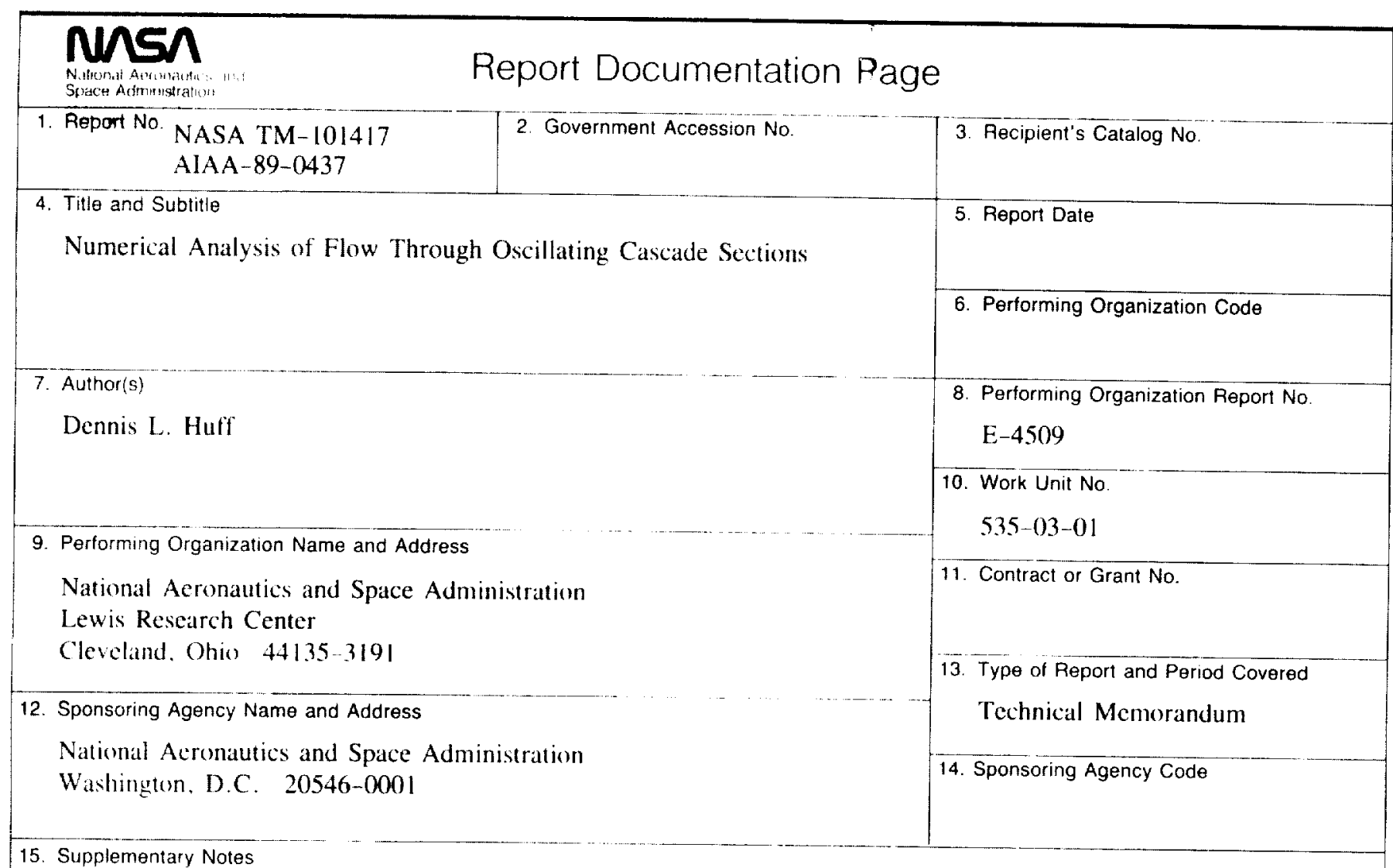

Prepared for the 27th Aerospace Sciences Meeting sponsored by the American Institute of Aeronautics and Astronautics, Reno, Nevada, January 9-12, 1989.

16. Abstracl

The design of turbomachinery blades requires the prevention of flutter for all operating conditions. However, flow field predictions used for aeroelastic analysis are not well understood for all flow regimes. The present research focuses on numerical solutions of the Euler and Navier-Stokes equations using an ADI procedure to model two-dimensional, transonic flow through oscillating cascades. The model preseribes harmonic pitching motions for the blade sections for both zero and nonzero interblade phase angles. The code introduces the use of a deforming grid technique for convenient specification of the periodic boundary conditions. Approximate nonreflecting boundary conditions have been coded for the inlel and exit boundary conditions. Sample unsteady solutions have been performed for an oscillating cascade and compared to experimental data. Also, test cases were run for a flat plate cascade to compare with the unsteady, small-perturbation, subsonic analysis. The predictions for oscillating cascades with nonzero interblade phase angles are in good agreement with experimental data and small-perturbation theory. The zero degree interblade phase angle cases, which were near a resonant condition, differ from the experiment and theory. The zero degree interblade phase angle cases, which were near a resonant condition, differ from the experiment and theory. Studies on reflecting versus nonreflecting inlet and exit boundary conditions show that the treatment of the boundary can have a significant effect on the first harmonic, unsteady pressure distributions for certain flow conditions. This code is expected to be used as a tool for reviewing simpler models that do not include the full nonlinear aerodynamics or as a final check for designs against flutter in turbomachinery.

17. Key Words (Suggested by Author(s))

Unsteady aerodynamics

Oscillating cascades

Viscous flow

18. Distribution Statement

Unclassified - Unlimited

Subject Category 02

Navier-Stokes equations

19. Security Classif. (of this report)

Unclassified

20. Security Classif. (of this page)

Unclassified
21. No of pages

24
22. Price*

A 0.3 


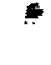


National Aeronautics and

Space Administration

\section{Lewis Research Center}

Cleveland. Ohio 44135

Onclad Bushese

Penally for Privato Uas $\$ 000$
SECOND CLASS MAIL

ADDRESS CORRECTION REQUESTEO

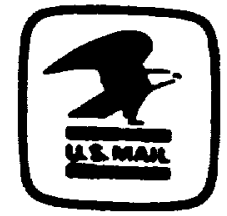

Poutage and Foes Pald National Aeronautics and Spece Administration NASA-451 